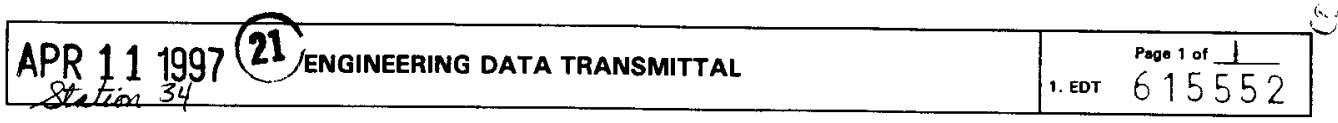

\begin{tabular}{|c|c|c|}
\hline $\begin{array}{l}\text { 2. To: (Receiving Organization) } \\
\text { Data Automation Engineering and } \\
\text { Services }\end{array}$ & $\begin{array}{l}\text { 3. From: (Originating Organization) } \\
\text { Facility Configuration Management and } \\
\text { Integration }\end{array}$ & 4. Related EDT No. \\
\hline $\begin{array}{l}\text { 5. Proj./Prog./Dept./Div.: } \\
\text { Facility Configuration Management } \\
\text { and Integration }\end{array}$ & $\begin{array}{l}\text { 6. Cog. Engr.: } \\
\text { J. B. Jech }\end{array}$ & $\begin{array}{l}\text { 7. Purchase Order No: : } \\
\text { N/A }\end{array}$ \\
\hline \multirow{2}{*}{\multicolumn{2}{|c|}{$\begin{array}{l}\text { 8. Originator Remarks: } \\
\text { Approval and release of MEL Phase I - System Design Description (SOD) }\end{array}$}} & $\begin{array}{l}\text { 9. Equip./Component No.: } \\
\text { N/A }\end{array}$ \\
\hline & & $\begin{array}{l}\text { 10. Systen/Bldg./Facility: } \\
\text { N/A }\end{array}$ \\
\hline \multirow{3}{*}{\multicolumn{2}{|c|}{ 11. Receiver Remarks: Design Baseline Document? []Yes [X]lio }} & $\begin{array}{l}\text { 12. Major Assm. Dwg. No.: } \\
\text { N/A }\end{array}$ \\
\hline & & $\begin{array}{l}\text { 13. Permit/Permit Application No.: } \\
\qquad N / A\end{array}$ \\
\hline & & $\begin{array}{l}\text { 14. Required Response Date: } \\
3 / 18 / 97\end{array}$ \\
\hline
\end{tabular}

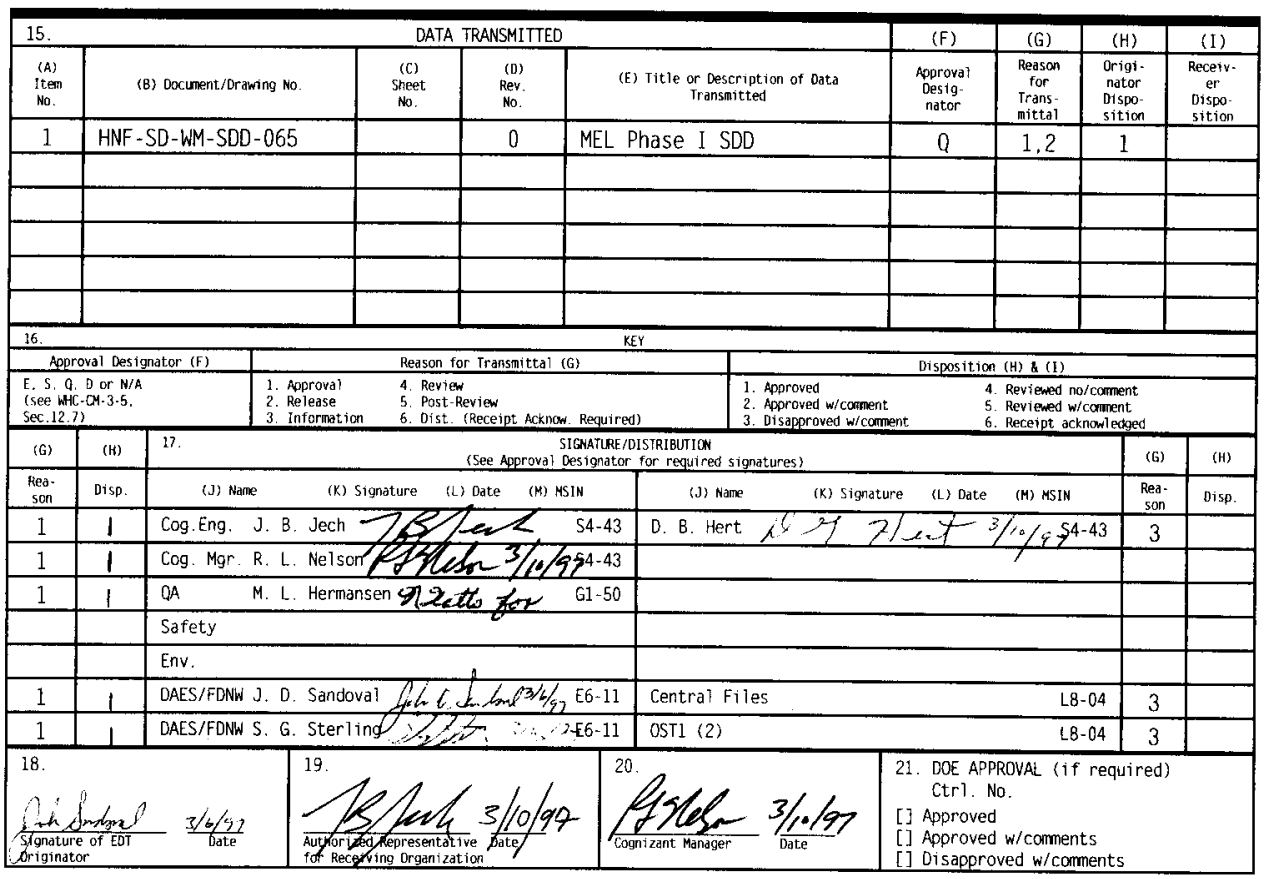

BD-7400-172-2 (04/94) GE,F097 
INSTRUCTIONS FOR COMPLETION OF THE ENGINEERING DATA TRANSMITTAL (USE BLACK INK OR TYPE)

\begin{tabular}{|c|c|}
\hline BLOCK & IITLE \\
\hline (11) ${ }^{*}$ & EDT \\
\hline (2) & To: (Receiving Organization) \\
\hline (3) & From: (Originating Organization) \\
\hline (4) & Related EOT No. \\
\hline$(5)^{*}$ & Proj./Prog./Dept./Div. \\
\hline$(6)^{*}$ & Cognizant Engineer \\
\hline 171 & Purchase Order No. \\
\hline (8) * & Originator Remarks \\
\hline (9) & Equipment/Component No. \\
\hline (10) & System/Bldg./Facility \\
\hline (11) & Receiver Remarks \\
\hline (12) & Major Assm. Dwg. No. \\
\hline (13) & Permit/Permit Applicstion No. \\
\hline 14) & Required $\mathrm{F}$ \\
\hline
\end{tabular}

- Pre-assigned EDT number.

- Enter the individual's name, title of the organization, or entity (e.g.. Distribution) that the EDT is being transmitted to.

- Enter the title of the org anization originating and transmitting the EDT.

- Enter EDT numbers which relate to the dats being tranemitted.

- Enter the Project/Program/Department/Division title or Project/Program scronym or Project Number, Work Order Number or Organization Code.

- Enter the name of the individual identified as being responsible for coordinating disposition of the EDT.

- Enter related Purchase Order (P.O.) Number, if available.

- Enter special or additional comments concerning transmittal, or "Key" retrieval words may be entered.

- Enter equipment/component number of affected item. if appropriate.

- Enter opplicable system, building or facility number, if appropriate.

- Enter special or additional comments concerring transmittat.

- Enter applicable drowing number of major assembly, if appropriate.

- Enter applicable permit or permit application number, if appropriote.

- Enter the date a response is required from individuals identified in Block 17 (Signature/Distribution).

(15) * Data Transmitted
(A) - Item Number
(B)* Document/Drawing No.
(C) * Sheet No.
(D). Rev. No.
(E) Title or Description of Data Transmitted
(F) Approval Designator
(G) Reason for Transmittal
(H) Originator Disposition
(I) Receiver Disposition

(16) KeY

- Enter sequential number, beginning with 1, of the information listed on EDT.

- Enter the unique identification number as signed to the document or drawing being transmitted.

- Enter the sheet number of the information being transmitted. If no sheet number, leave blank.

- Enter the revision number of the information being tramsmitted. If no revision number, leave blank.

- Enter the title of the document or drawing or a brief description of the subject if no title is identified.

- Enter the appropriate Approvaf Designator (Block 15). Also, indicate the appropriate approvals for each item listed, h.e., SQ, ESO, otc.

- Enter the appropriate code to identify the purpose of the data transmittal (see Block 16).

- Enter the appropriate disposition code (see Block 16).

- Enter the appropriate disposition code (see Block 16).

- Number codes used in completion of Blocks 15 (G), (H), and (I), and 17 (G), (H) (Signature/Distribution).

(17) Signsture/Distribution
(G) Reason
(H) Disposition
(J) Name
(K)* Signature
(L) * Date
(M) * MSIN

118] Signature of EDT Originator

(19) Authorized Representative for Receiving Organization

(20) * Cognizant Manager

(21)* DOE Approval

- Enter the code of the reason for transmittal (Block 16).

- Enter the code for the disposition (Block 16).

- Enter the signature of the individual completing the Disposition $17(\mathrm{H})$ and the Transmittal.

- Obtain appropriate signature(s).

- Enter dete signature is obtained.

- Enter MSIN. Note: If Distribution Sheet is used, show entire distribution fincluding that indicated on Poge 1 of the EDTh on the Distribution Sheet.

- Enter the signature and date of the individual originating the EDT lentered prior to transmittal to Receiving Organization). It the EDT originator is the cognizant engineer, sign both Blocks 17 and 18 .

- Enter the signature and date of the individual identified by the Receiving Organization as autherized to approve disposition of the EDT and ecceptance of the dete transmitted, as applicable.

- Enter the signature and date of the cognizant mansger. (This signature is authorization for release.)

- Enter DOE epproval (if required) by eignature or control number thet tracks the approval to a signature. and indicate DOE action.

TAsterick denote the required minimum items check by Configuration Documentation prior to release: these are the minimum release requirements. 


\section{System Design Description For Master Equipment List, Phase I}

\section{J. D. Sandoval}

Fiuor Daniel Northwest

P. 0. Box 1050

Richland. WA 99352

U.S. Department of Energy Contract DE-AC06-87RL10930

EDT/ECN: 615552

Org Code: 77820

B\&R Code: EW3120071
UC: 605

Charge Code: N12A4

Total Pages: $10 \sigma^{\circ} 107$

$\mathrm{kN}$

Key Words: System Design Description

Abstract: Approval and release for the Master Equipment List (MEL) Phase I, for the System Design Description (SDD)

TRADEMARK DISCLAIMER. Reference herein to any specific commercial product, process, or service by trade name, trademark, manufacturer, or otherwise, does not necessarily constitute or imply its endorsement, recommendation, or favoring by the United States Government or any agency thereof or its contractors or subcontractors.

Printed in the United States of America. To obtain copies of this document, contact: WHC/BCS Document Control Services. P.O. Box 1970. Mailstop H6-08. Richland WA 99352. Phone (509) 372-2420; Fax (509) 376-4989
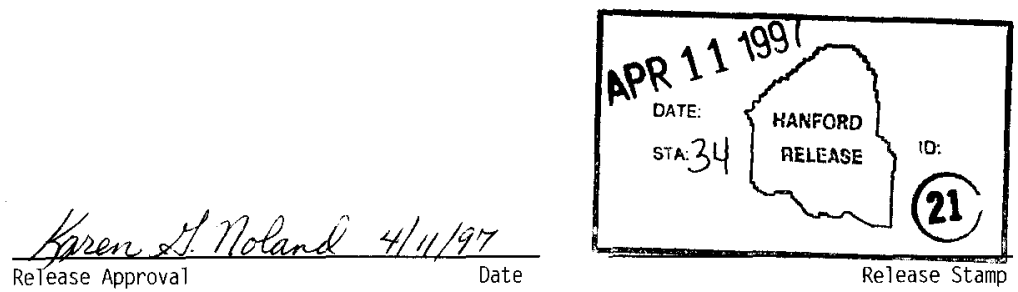

\section{Approved for Public Release}


THIS PAGE INTENTIONALLY LEFT BLANK 
HNF-SD-WM-SDD-065

Revision 0

Page 1

\section{SYSTEM DESIGN DESCRIPTION}

\section{FOR}

\section{MASTER EQUIPMENT LIST PHASE I}

Revision 0

\section{Prepared by:}

Data Automation Engineering \& Services Fluor-Daniel Northwest, Inc.

November 1996

Prepared for:

Lockheed Martin Hanford Corporation

PO Box 1500

Richland, Washington 99352-1505 
HNF-SD-WM-SDD-065

Revision 0

Page 2

\section{TABLE OF CONTENTS}

PAGE

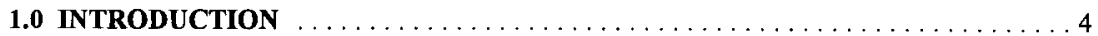

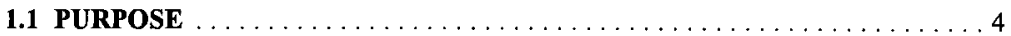

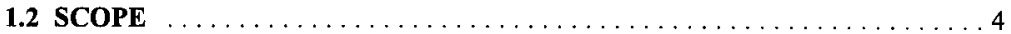

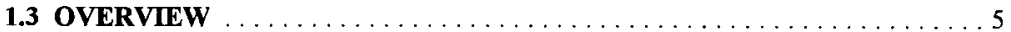

1.4 DEFINITIONS AND ABBREVIATIONS $\ldots \ldots \ldots \ldots \ldots \ldots \ldots$

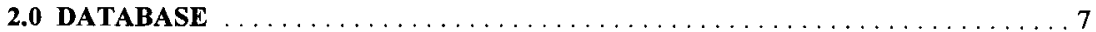

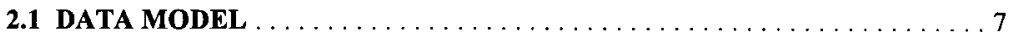

2.2 ENTITY RELATIONSHIP DIAGRAM $\ldots \ldots \ldots \ldots \ldots \ldots \ldots$

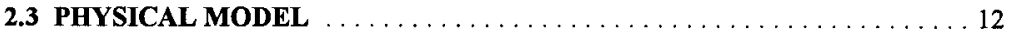

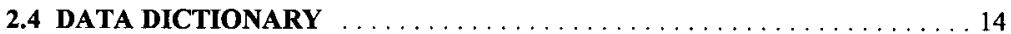

2.4.1 USER INTERFACE TABLES $\ldots \ldots \ldots \ldots \ldots \ldots \ldots \ldots \ldots$

2.4.2 SYSTEM TABLES $\ldots \ldots \ldots \ldots \ldots \ldots \ldots \ldots \ldots \ldots \ldots$

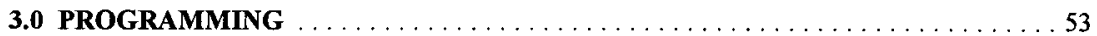

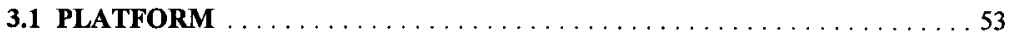

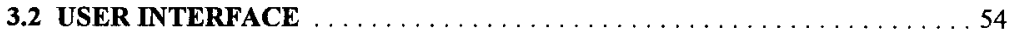

3.2.1 PROGRAM UNITS $\ldots \ldots \ldots \ldots \ldots \ldots \ldots \ldots \ldots \ldots \ldots \ldots \ldots$

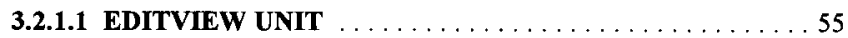

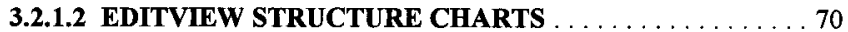

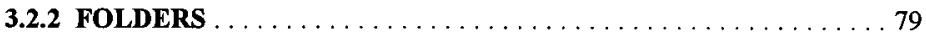

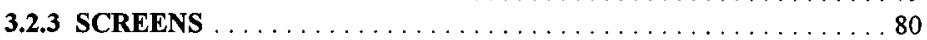

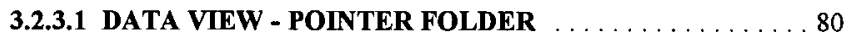

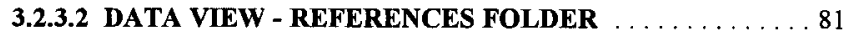

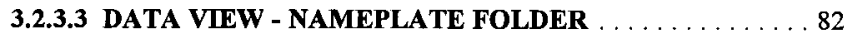

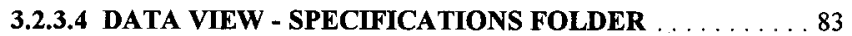

3.2.3.5 DATA VIEW - SAFETY FOLDER $\ldots \ldots \ldots \ldots \ldots \ldots 84$

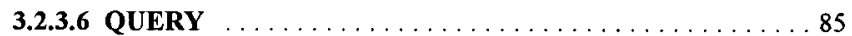

3.2.3.7 REPORTS . . . . . . . . . . . . . . . . . . . . 86

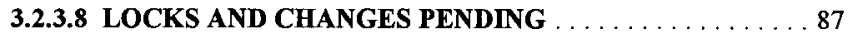

3.2.3.9 CREATE NEW EINS . . . . . . . . . . . . . . . . 88

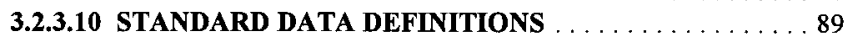

3.2.3.11 CHANGE LOG $\ldots \ldots \ldots \ldots \ldots \ldots \ldots \ldots$

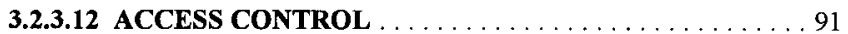

3.2.3.13 ABOUT $\ldots \ldots \ldots \ldots \ldots \ldots \ldots \ldots \ldots \ldots \ldots \ldots \ldots$ 
HNF-SD-WM-SDD-065

Revision 0

Page 3

3.2.4 MENUS $\ldots \ldots \ldots \ldots \ldots \ldots \ldots \ldots \ldots \ldots \ldots \ldots \ldots \ldots \ldots \ldots$

3.2.4.1 MANN MENU $\ldots \ldots \ldots \ldots \ldots \ldots \ldots \ldots \ldots \ldots \ldots \ldots$

3.2.4.2 QUERY MENU $\ldots \ldots \ldots \ldots \ldots \ldots \ldots \ldots \ldots \ldots . \ldots 94$

3.2.4.3 LOCKS AND PENDING CHANGES MENU _... . . . 94

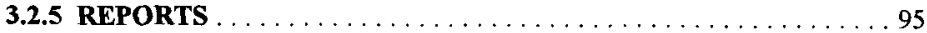

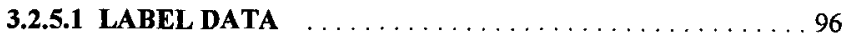

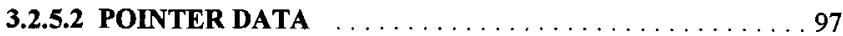

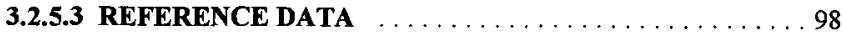

3.2.5.4 NAMEPLATE DATA $\ldots \ldots \ldots \ldots \ldots \ldots \ldots \ldots 9 . \ldots \ldots$

3.2.5.5 SPECIFICATION DATA $\ldots \ldots \ldots \ldots \ldots \ldots \ldots \ldots$

3.2.5.6 SAFETY DATA $\ldots \ldots \ldots \ldots \ldots \ldots \ldots \ldots \ldots, 101$

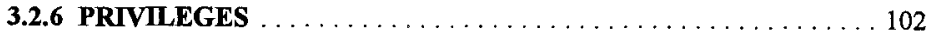

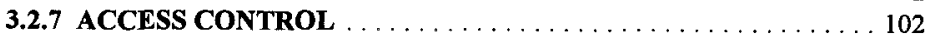

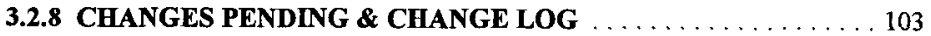

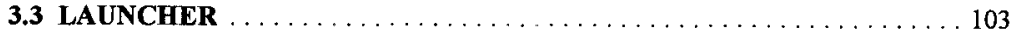

3.4 DISTRIBUTION / INSTALLATION $\ldots \ldots \ldots \ldots \ldots \ldots \ldots \ldots \ldots \ldots$

3.4.1 RELEASE AREAS \& VERSIONS $\ldots \ldots \ldots \ldots \ldots \ldots \ldots \ldots$

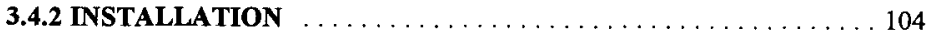

3.4.3 USER COMPUTER RESOURCE REQUIREMENTS . . . . . 105

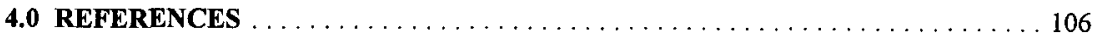


HNF-SD-WM-SDD-065

Revision 0

Page 4

\section{SYSTEM DESIGN DESCRIPTION FOR \\ MASTER EQUIPMENT LIST PHASE I}

\subsection{INTRODUCTION}

\subsection{PURPOSE}

This System Design Description (SDD) is for the Master Equipment List Phase I (MEL). It has been prepared following the WHC-CM-3-10, "Software Practices," (Ref. 6). This SDD describes the internal design for implementation of the MEL Phase $I$.

\subsection{SCOPE}

The MEL Phase I application includes features to;

- Enable users to view a variety of information about equipment in various data tables using a 'folder' metaphor.

o Provide data search features to quickly locate information about the equipment.

o Provide query functions for locating key data about the equipment.

o Provide the ability to generate standard reports of folder information.

- Maintain MEL Phase I user IDs and passwords.

o Provide the ability to edit existing data, enter new data, and delete data based upon predefined privileges to do so.

- Provide audit trail information for monitoring and logging data entry and change. 
HNF-SD-WM-SDD-065

Revision 0

Page 5

\subsection{OVERVIEW}

The remainder of this SDD is divided into four main sections.

Section 2, "Database" contains data models, entity relationship diagrams, physical models, and a data dictionary.

Section 3, "Programming" contains platform information, screen views of the user interface, startup and execution parameter definitions, and detailed information regarding installation.

The final section, Section 4, "References", contains the references for this document.

\subsection{DEFINITIONS AND ABBREVIATIONS}

The following are definitions of abbreviations and terms used in this document:

$\begin{array}{ll}\text { ASCII } & \text { American Standard Code for Information Interchange } \\ \text { DAES } & \text { Data Automation Engineering \& Services } \\ \text { DCA } & \text { Data Collection Application } \\ \text { ERD } & \text { Entity Relationship Diagram } \\ \text { EP } & \text { Engineering Practices } \\ \text { MEL Phase I } & \text { Master Equipment List Phase I } \\ \text { HLAN } & \text { Hanford Local Area Network } \\ \text { PC } & \text { Personal Computer } \\ \text { SDD } & \text { System Design Description } \\ \text { SRS } & \text { System Requirements Specification } \\ \text { STD } & \text { State Transition Diagram } \\ \text { UA } & \text { User Application }\end{array}$

ASCII--A standard used to represent text and control characters in a computer environment. The ASCII format relates hexadecimal numbers to these text and control characters.

Engineering Practices (EPs)--supporting document management manual WHC-CM-6-1 that establishes the manner and order for performance of common configuration management practices relating to engineering tasks. 
HNF-SD-WM-SDD-065

Revision 0

Page 6

Entity--subpiece, recognizable, with a distinct function within the MEL Phase I project.

ERD--diagram showing system/environment entities and relationships.

MEL Phase I--The project being performed by DAES to provide a means of locating and querying information about equipment in Tank Farms. See WHC-SD-WM-SDP-012 (Ref. 4) for the work plan for this system.

Approval Designator--defined in WHC-CM-3-5, "Document Control and Records Management Manual," Section 12.7 (Ref. 7).

Module--subsystem of the MEL Phase I system that encompasses attributes of the system including hardware and software.

STD--diagram showing control sequence for the system/environment.

System Design Description--documentation of essential and implementation design that gives descriptions of entities and attributes of the design.

System Requirements Specification--the documentation of essential requirements (functions, performance, design constraints and attributes) of the computer software and its external interfaces. 
HNF-SD-WM-SDD-065

Revision 0

Page 7

2.0 DATABASE

2.1 DATA MODEL

The following data model show the relationships of the data for the MEL. 
HNF-SD-WM-SDD-065

Revision 0

Page 8

- Roctengles reprosont entities.

- Rounded rectanglos roprosent rolationthips.

Pont cardinglity.

- Connectors with ' $x, x$ numbors roprosont Cardinaling' and 'Mastor EIN' gro read as followi:

"Pointer Data ie lentified by 1 to 1 (only 1) Mastor EIN-

and -A Master EIN uniquoly lototifios $010 \mathrm{~m}$ (many) Pointer Data"
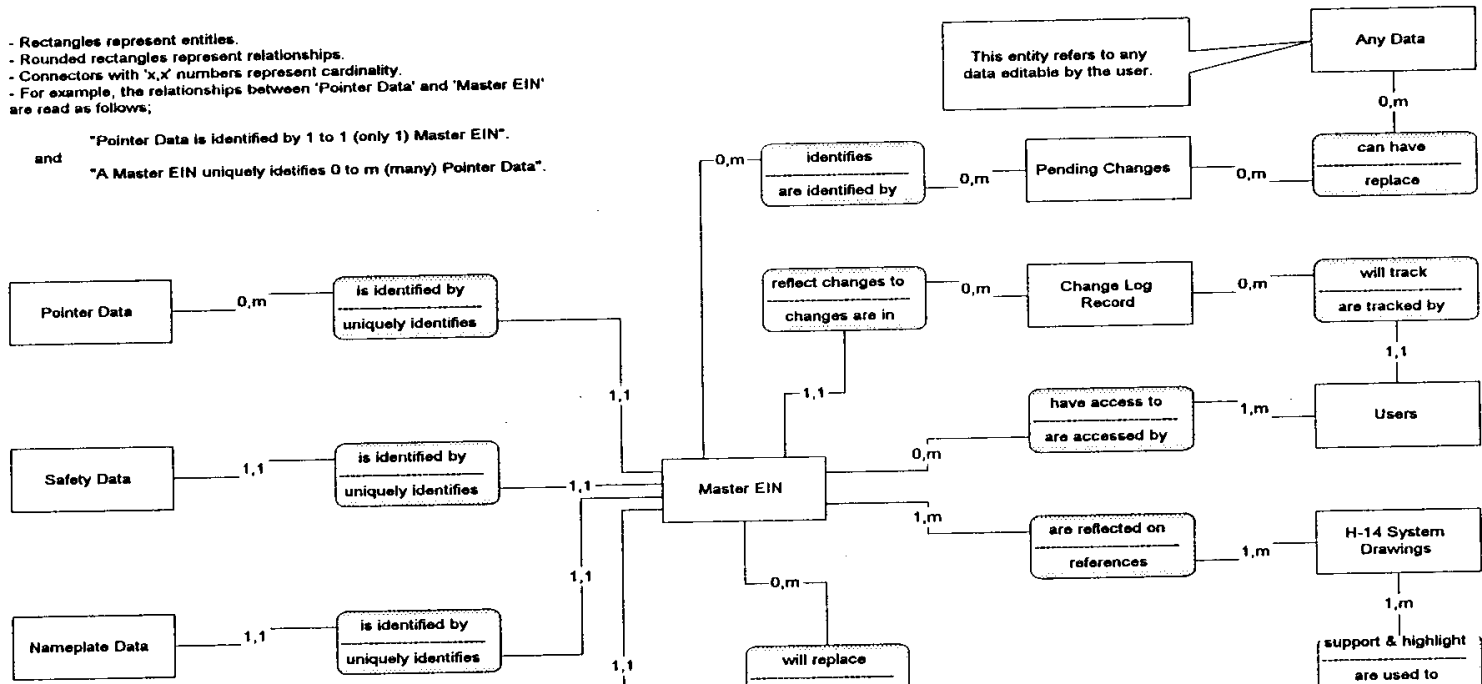

Spocification Data

$1,1-\frac{\text { is identifiod by }}{\text { uniqualy identifies }}$


HNF-SD-WM-SDD-065

Revision 0

Page 9

\subsection{ENTITY RELATIONSHIP DIAGRAM}

The following entity relationship diagrams show the relationships of the MEL. 
HNF-SD-WM-SDD-065

Revision 0

Page 10

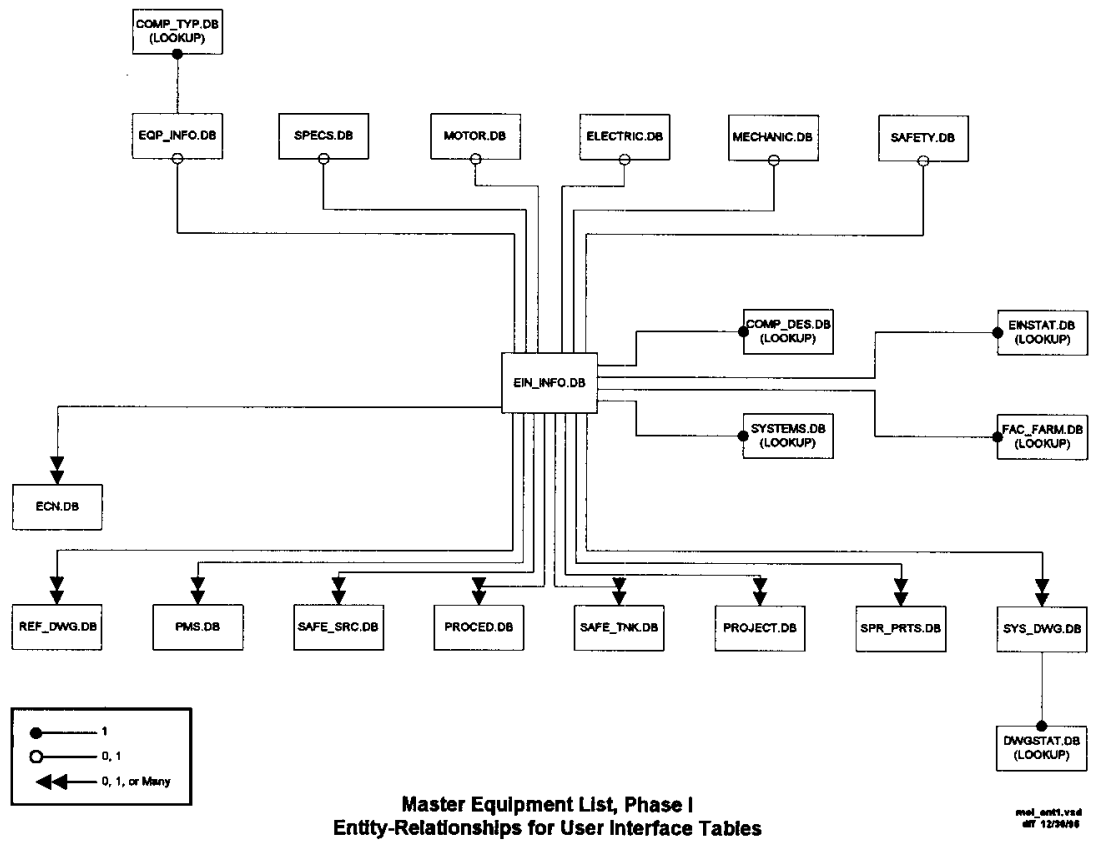


HNF-SD-WM-SDD-065

Revision 0

Page 11

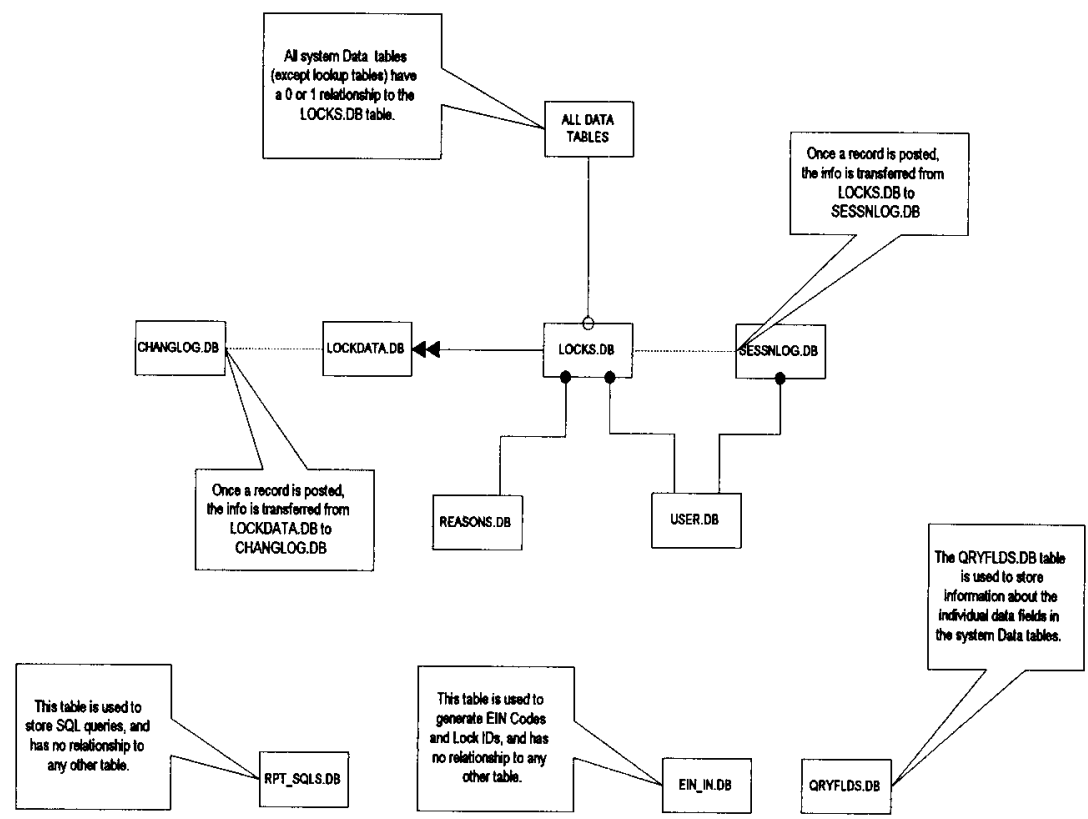

$$
\begin{aligned}
& 0-1 \\
& 0-0.1 \\
& 4-0,1, \text { or Many }
\end{aligned}
$$

Master Equipment List, Phase I Entity-Relationships for System Tables 
HNF-SD-WM-SDD-065

Revision 0

Page 12

\subsection{PHYSICAL MODEL}

The following physical model diagram shows the data and lookup tables for the MEL Phase I and field links. 
HNF-SD-WM-SDD-065

Revision 0

Page 13

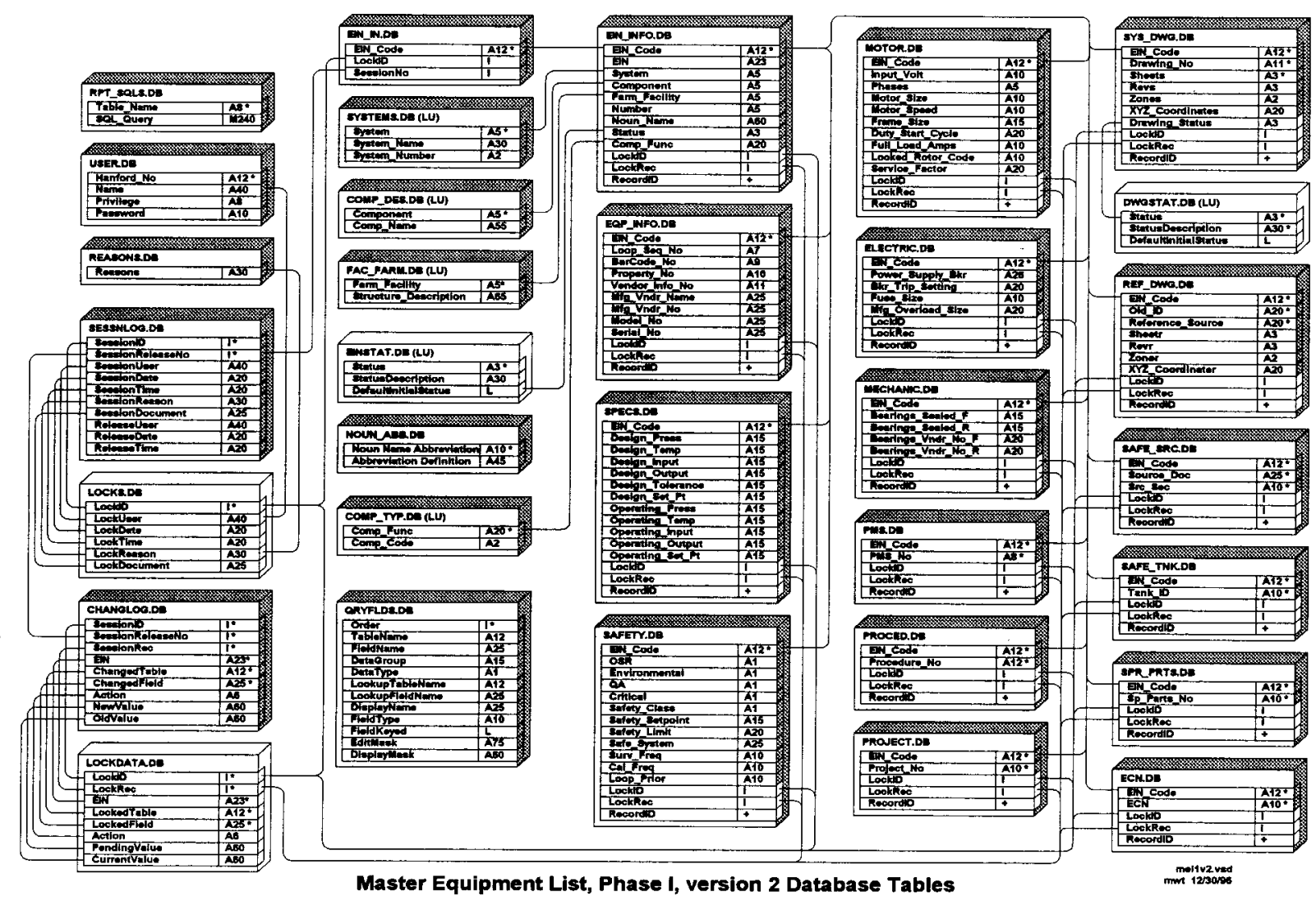



HNF-SD-WM-SDD-065

Revision 0

Page 14

\subsection{DATA DICTIONARY}

This Data Dictionary (DD) provides a detailed description of the data structures and their elements which will compose the MEL Phase I application. The DD does not define explicit relationships between structure entities - those are better illustrated in Section 2.2 "Entity Relationship Diagram" of this document.

This Data Dictionary is organized into two major parts;

o Structures that support the user interface and the data gathered in its use,

- Structures that support the system as a whole - including tables used during reports generation, change log tables, session tables, and general system support tables.

These parts each contain a list of table structures. Because the number of these structures is relatively small, they are presented in the order that follows their application to the MEL Phase I application. An alphabetical ordering does not seem useful for this application.

Each structure is divided into its component data elements or fields. Fields are listed in the order that presents key fields first (as required in a Paradox implementation). Each field will be described in terms of:

- Field Name

The field's reference name.

- Type \& Size The data type and size (see description below).

- Key

- Required

- Example

- Restriction An asterisk $\left(^{*}\right)$ next to the type \& size indicates a key field. Data that is required (per customer requirement or design) is indicated by 'True'.

- Description Example of a typical data entry.

Any special restrictions or conditions regarding the data. Textual description of the data.

Type and size formats are typically shown with the shorthand of a single character type which is sometimes followed by a number indicating the number of bytes reserved to hold the data value. The types are defined as:
$A=$ Alphanumeric (with limited reserved space)
$\mathrm{N}=$ Numeric (floating point)
$I=$ Long integer (4 bytes)
$\mathrm{S}=$ Small integer $(2$ bytes)
$\mathrm{D}=$ Date (Paradox internal format) 
HNF-SD-WM-SDD-065

Revision 0

Page 15

M = Memo (with limited reserved base table space and virtually unlimited memo table space)

$\mathbf{L}=$ Logical (boolean value, True or False)

$+=$ Autoincrement (small integer value, incremented automatically with each new record)

Alphanumeric (A) formats always include a number - e.g. A25 represents a 25 byte alphanumeric data field. In this example, 25 bytes are reserved in the table for each record.

For memos (M), the number represents the bytes reserved in the base table (the named table structure) although the full memo length is virtually unlimited with the use of an auxiliary memo table. The number is important since a form field will display the memo data I the base table without accessing the memo table (this saves time). The memo table is accessed only when the complete memo is required. Typically, form fields should be sized to match the base size of the memo - e.g., M120 represents a memo field of 120 bytes immediately accessible in the base table even though the actual memo length is as long as necessary to contain the complete memo data.

Restriction formats are usually made up of alphanumeric characters that represent certain values. The types are defined as:

$\mathrm{a}=$ any alphanumeric character

$\mathrm{n}=$ Number (0-9)

[] = Any character(s) surrounded by brackets are optional.

Lookup $=$ The value must be selected from a pre-defined list.

A restriction format may be made up of a combination of alphanumeric characters, numbers, and literal values. For example, a restriction format for a H-14 drawing would be represented by the following:

\section{H-14-nnnnnn}

Therefore, H-14-012345, would be considered a valid value, while H-14-ABC123 would not. 


\subsubsection{USER INTERFACE TABLES}

EIN_INFO.DB This table identifies all the EINs entered.

EIN Code

Size \& Type: A12*

Required: False

Folder:

Example: EINO00000033

Restrictions:

Information: This record identifier will be generated automatically by the system. It is a counter used to grant a unique ID for each EIN record.

EIN

Size \& Type: A23

Required: True

Folder: Equipment Identification Number block.

Example: ANO00-AS-AOD-1001

Restrictions: aa[aaaa]-aa[aaa]-aa[aaa]-nnn[a]

Information: This field is a concatenation of the Farm Facility, System, Component, and Number fields. It is generated automatically by the system.

System

Size \& Type: A5

Required: True

Folder: Equipment Identification Number block.

Example: CHMA

Restrictions: lookup

Information: This field forces a value from the Systems.DB, System field.

Component

Size \& Type: A5

Required: True

Folder: Equipment Identification Number block.

Example: AOD

Restrictions: Lookup

Information: This field forces a value from the Comp_Des.DB, Component field.

Farm_Facility

Size \& Type: A6

Required: True

Folder: Location block.

Example: $\quad$ ANOO0

Restrictions: Lookup

Information: This field forces a value from the Fac_Farm.DB, Farm_Facility field. 
HNF-SD-WM-SDD-065

Revision 0

Page 17

Number

Size \& Type: A4

Required: True

Folder: Equipment Identification Number block.

Example: 1001

Restrictions: $\mathrm{nnn}[\mathrm{na}]$

Information: This field is the unique part of the EIN.

Noun Namel

Size \& Type: A32

Required: True

Folder: Equipment Identification Number block.

Example: XMITTER

Restrictions: Lookup

Information: This field provides a lookup to noun names from similar EINs. However, a typed value may be entered.

Noun Name2

Size \& Type: A32

Required: True

Folder: $\quad$ Equipment Identification Number block.

Example: XMITTER

Restrictions: Lookup

Information: This field provides a lookup to noun names from similar EINs. However, a typed value may be entered.

Status

Size \& Type: A3

Required:

Folder: Equipment Identification Number block.

Example: 1

Restrictions:

Information: This field provides a lookup to number representing a status.

Comp_Func

Size \& Type: A20

Required: True

Folder: $\quad$ Equipment Identification Number block.

Example: HORIZONTAL

Restrictions: lookup

Information: This field is used as a link to the Comp_Typ.DB, Comp_Func field. This is a lookup value that forces a selection from the list. 
LockID

Size \& Type: I

Required:

Folder:

Example: 1

Restrictions:

Information: This is the identifier for the lock session. This data is used internally by the system.

LockRec

Size \& Type: I

Required:

Folder:

Example: 1

Restrictions:

Information: This is the identifier for the record being modified. This data is used internally by the system.

RecordID

Size \& Type: +

Required:

Folder:

Example: 1

Restrictions:

Information: This is a record identifier used internally by the system.

EQP_INFO.DB This table contains the equipment information for each EIN entered.

EIN_Code

Size \& Type: A12*

Required: True

Folder:

Example: EINO00000033

Restrictions:

Information: This record identifier will be generated automatically by the system. It is a counter used to grant a unique ID for each EIN record.

Loop_Seq_No

Size \& Type: A7

Required:

Folder: Pointer

Example: AN212-1

Restrictions: aannn-n

Information: This field is used as the key field in CBRS (Component Based Recall System). This is the reference source for historical data. 
HNF-SD-WM-SDD-065

Revision 0

Page 19

BarCode_No

Size \& Type: A9

Required:

Folder: Pointer

Example: S000120WT

Restrictions:

Information: Barcode Number. Unique identifier assigned to the equipment that resides/functions in the EIN Location, used for developing equipment history information (cradle to grave tracking) versus functional location history information.

Property_No

Size \& Type: A10

Required:

Folder: Pointer

Example: WC42029

Restrictions:

Information: Property Number. Unique identifier (Barcode) that property assigns to equipment for tracking purposes in the Property Management System.

Vendor_Info_No

Size \& Type: A11

Required:

Folder: Pointer

Example: $\quad 0022574-004$

Restrictions: nnnnnnn-nnn

Information: Vendor Information Number. Used as the key field in the Certified Vendor Information System (Soft Reporting).

Mfg_Vndr_Name

Size \& Type: A25

Required:

Folder: Pointer

Example: Ingersoll-Rand

Restrictions:

Information: Manufacturer or Vendor Name.

Mfg_Vndr_No

Size \& Type: A25

Required:

Folder: Pointer

Example: $\quad 37 \mathrm{~F} 427 \mathrm{X} 484$

Restrictions:

Information: Manufacturer or Vendor Number. Unique identifier Manufacturer/Vendor assigns to equipment. 
HNF-SD-WM-SDD-065

Revision 0

Page 20

Model No

Size \& Type: A25

Required:

Folder: Pointer

Example: $\quad 37 F 427 \times 484$

Restrictions:

Information: Model Number. Unique identifier manufacturer assigns to equipment.

Serial_No

Size \& Type: A25

Required:

Folder: Pointer

Example: $\quad 30 \mathrm{~T}-714984$

Restrictions:

Information: Serial Number. Unique identifier that Manufacturer assigns to equipment.

LockID

Size \& Type: I

Required:

Folder:

Example: 1

Restrictions:

Information: This is the identifier for the lock session. This data is used internally by the system.

LockRec

Size \& Type: I

Required:

Folder:

Example: 1

Restrictions:

Information: This is the identifier for the record being modified. This data is used internally by the system.

RecordID

Size \& Type: +

Required:

Folder:

Example: 1

Restrictions:

Information: This is a record identifier used internally by the system.

SPECS.DB This table contains the specification information for each EIN as applicable.

EIN_Code

Size \& Type: A12*

Required: True

Folder:

Example: EINO00000033

Restrictions: 
HNF-SD-WM-SDD-065

Revision 0

Page 21

Information: This record identifier will be generated automatically by the system. It is a counter used to grant a unique $\mathrm{ID}$ for each EIN record.

\section{Design_Press}

Size \& Type: A15

Required:

Folder: Specifications

Example: PSI

Restrictions:

Information: Design Pressure Range. Typical units of measure in the industry.

\section{Design_Temp}

Size \& Type: Als

Required:

Folder: Specifications

Example: $\quad 80-100 \mathrm{~F}$

Restrictions:

Information: Design Temperature Range. Fahrenheit will be the unit of measure.

\section{Design_Input}

Size \& Type: A15

Required:

Folder: Specifications

Example: $\quad$ gpm

Restrictions:

Information: Design Input Range.

\section{Design_Output}

Size \& Type: A15

Required:

Folder: Specifications

Example: pph

Restrictions:

Information: Design Output Range.

Design_Tolerance

Size \& Type: A15

Required:

Folder: Specifications

Example: $\quad 23 \%$

Restrictions:

Information: Design Accuracy. Units could be $\%$ or ( $+/-)$. 


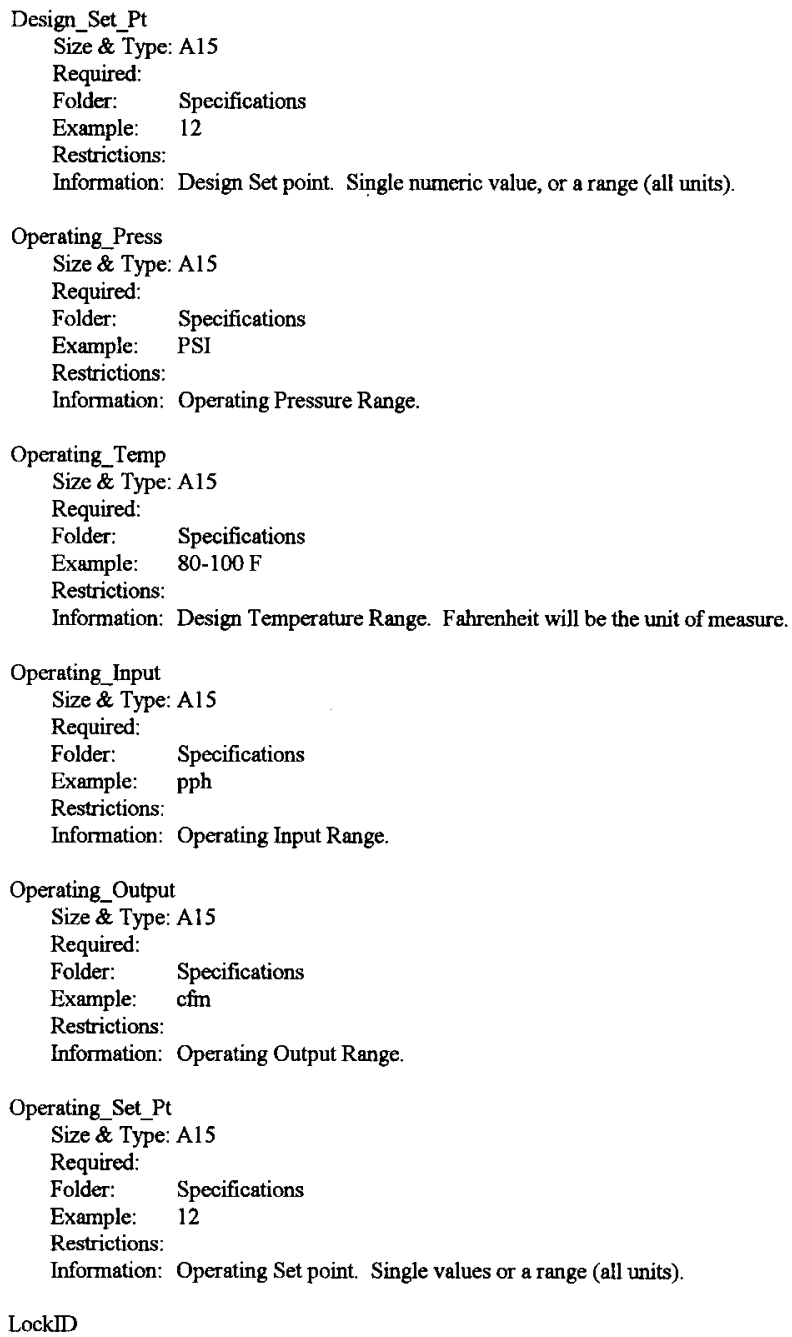


HNF-SD-WM-SDD-065

Revision 0

Page 23

Size \& Type: I

Required:

Folder:

Example: 1

Restrictions:

Information: This is the identifier for the lock session. This data is used internally by the system.

LockRec

Size \& Type: I

Required:

Folder:

Example: 1

Restrictions:

Information: This is the identifier for the record being modified. This data is used internally by the system.

RecordID

Size \& Type: +

Required:

Folder:

Example: 1

Restrictions:

Information: This is a record identifier used internally by the system.

SAFETY.DB This table contains the safety information for each EIN as applicable.

EIN_Code

Size \& Type: A12*

Required: True

Folder:

Example: EIN000000033

Restrictions:

Information: This record identifier will be generated automatically by the system. It is a counter used to grant a unique ID for each EIN record.

OSR

Size \& Type: Al

Required: True

Folder: Classification block.

Example: $\quad \mathrm{Y}$

Restrictions: $\mathrm{Y}$ or $\mathrm{N}$ Information:

This field is a boolean value represented by either a $\mathrm{Y}$ (Yes) or $\mathrm{N}$ (No).

Environmental

Size \& Type: Al

Required: True

Folder: $\quad$ Classification block.

Example: $\quad Y$

Restrictions: $\mathrm{Y}$ or $\mathrm{N}$

Information: This field is a boolean value represented by either a $\mathrm{Y}$ (Yes) or $\mathrm{N}(\mathrm{No})$. 
HNF-SD-WM-SDD-065

Revision 0

Page 24

QA

Size \& Type: Al

Required:

Folder: Classification block.

Example: $\quad \mathrm{Y}$

Restrictions: $\mathrm{Y}$ or $\mathrm{N}$

Information: This field is a boolean value represented by either a $\mathrm{Y}$ (Yes) or $\mathrm{N}(\mathrm{No})$.

Critical

Size \& Type: A1

Required: False

Folder: $\quad$ Safety

Example: $\quad \mathrm{Y}$

Restrictions: $\mathrm{Y}, \mathrm{N}, 1,2,3,4$

Information: This is the critical classification.

Safety_Class

Size \& Type: A1

Required: True

Folder: Safety

Example: $\quad Y$

Restrictions: $Y, N, 1,2,3,4$

Information: This is the safety classification.

Safety_Setpoint

Size \& Type: Al5

Required:

Folder: Safety

Example:

Restrictions:

Information: Safety set point determination.

Safety Limit

Size \& Type: A20

Required:

Folder: Safety

Example:

Restrictions:

Information: Safety limit determination.

Safe_System

Size \& Type: A25

Required:

Folder: Safety

Example:

Restrictions:

Information: Safety system. 
HNF-SD-WM-SDD-065

Revision 0

Page 25

Surv_Freq

Size \& Type: A10

Required:

Folder: Safety

Example: Weekly

Restrictions

Information: Surveillance Frequency. Frequency at which equipment is inspected.

Cal_Freq

Size \& Type: AI0

Required:

Folder: Safety

Example: Weekly

Restrictions:

Information: Calibration Frequency. Frequency at which the equipment is calibrated.

Loop_Prior

Size \& Type: A10

Required:

Folder: Safety

Example:

Restrictions:

Information: Loop priority.

LockID

Size \& Type: I

Required:

Folder:

Example: 1

Restrictions:

Information: This is the identifier for the lock session. This data is used internally by the system.

LockRec

Size \& Type: I

Required:

Folder:

Example: 1

Restrictions:

Information: This is the identifier for the record being modified. This data is used internally by the system.

RecordID

Size \& Type: +

Required:

Folder:

Example: 1

Restrictions:

Information: This is a record identifier used internally by the system. 
MOTOR.DB This table contains the motor information for each EIN as applicable.

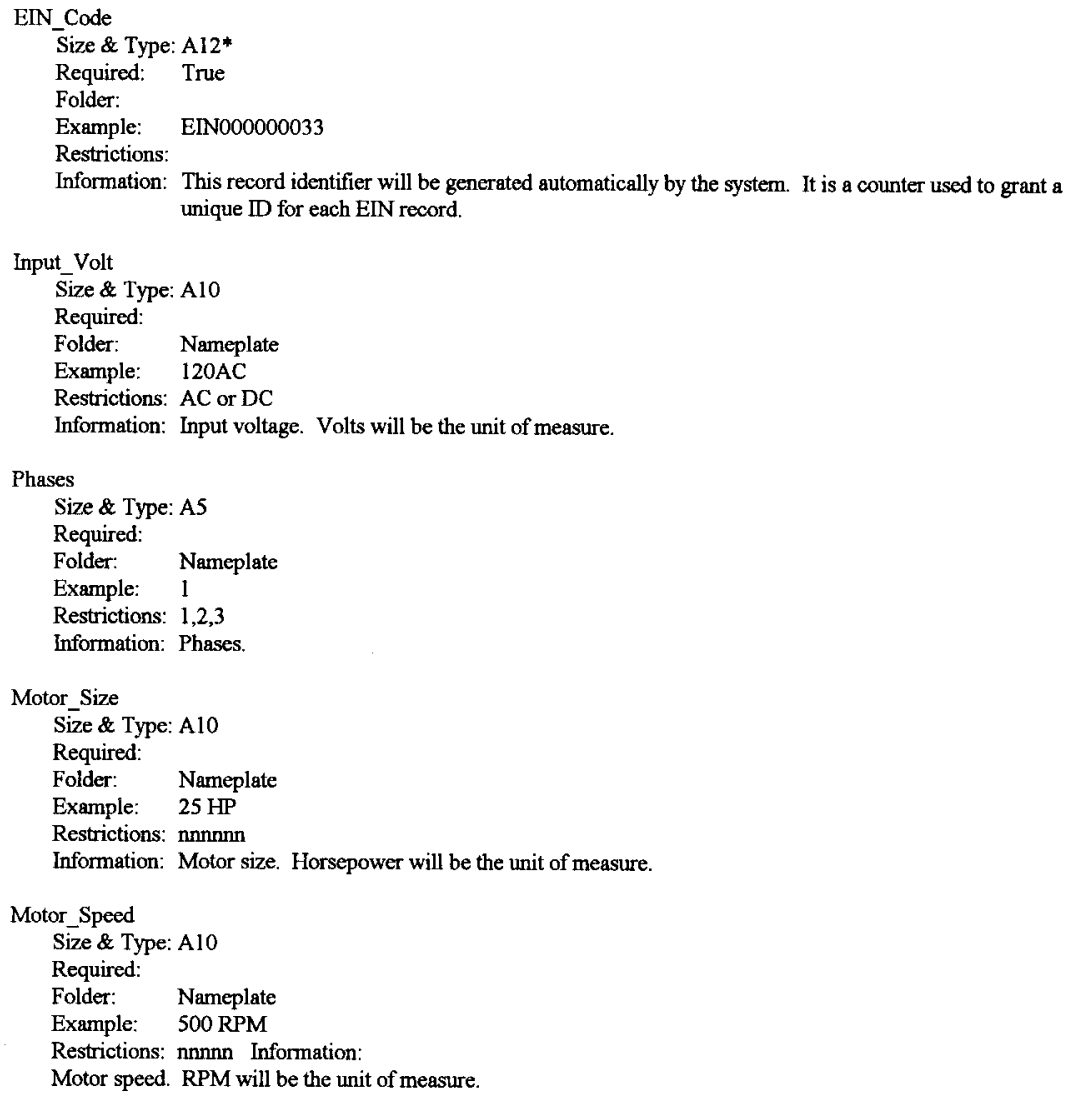


HNF-SD-WM-SDD-065

Revision 0

Page 27

Frame Size

Size \& Type: A15

Required:

Folder: Nameplate

Example: $284 \mathrm{~T}$

Restrictions:

Information: Frame size.

Duty_Start_Cycle

Size \& Type: A20

Required:

Folder: Nameplate

Example:

Restrictions:

Information: Duty Start Cycle.

Full Lood Amps

Size \& Type: A10

Required:

Folder: Nameplate

Example: 12

Restrictions: $\mathrm{nn}$

Information: Full Load Amps. AMPS will be the unit of measure.

Locked_Rotor_Code

Size \& Type: A10

Required:

Folder: Nameplate

Example:

Restrictions:

Information: Locked Rotor Code.

Service_Factor

Size \& Type: A20

Required:

Folder: Nameplate

Example: .5

Restrictions: $0<n<1$

Information: Design Service Factor. No unit of measure.

Lock3D

Size \& Type: I

Required:

Folder:

Example: 1

Restrictions:

Information: This is the identifier for the lock session. This data is used internally by the system. 
HNF-SD-WM-SDD-065

Revision 0

Page 28

LockRec

Size \& Type: I

Required:

Folder:

Example: 1

Restrictions:

Information: This is the identifier for the record being modified. This data is used internally by the system.

RecordID

Size \& Type: +

Required:

Folder:

Example: 1

Restrictions:

Information: This is a record identifier used internally by the system.

ELECTRIC.DB This table contains the motor information for each EIN as applicable.

EIN Code

Size \& Type: A12*

Required: True

Folder:

Example: EIN000000033

Restrictions:

Information: This record identifier will be generated automatically by the system. It is a counter used to grant a unique ID for each EIN record.

Power_Supply_Bkr

Size \& Type: A26

Required:

Folder: Nameplate

Example:

Restrictions:

Information: Power Supply Breaker.

Bkr_Trip_Setting

Size \& Type: A20

Required:

Folder: Nameplate

Example: 100 AMPS

Restrictions:

Information: Breaker Trip Setting. AMPS will be the unit of measure. 
HNF-SD-WM-SDD-065

Revision 0

Page 29

\section{Fuse_Size}

Size \& Type: A10

Required:

Folder: Nameplate

Example: 100 AMPS

Restrictions:

Information: Fuse Size. AMPS will be the unit of measure.

Mfg_Overload_Size

Size \& Type: A20

Required:

Folder: Nameplate

Example:

Restrictions:

Information: Manufacturer Overload Size.

LockID

Size \& Type: I

Required:

Folder:

Example:1 1

Restrictions:

Information: This is the identifier for the lock session. This data is used internally by the system.

LockRec

Size \& Type: I

Required:

Folder:

Example: 1

Restrictions:

Information: This is the identifier for the record being modified. This data is used internally by the system.

RecordID

Size \& Type: +

Required:

Folder:

Example: 1

Restrictions:

Information: This is a record identifier used internally by the system. 
MECHANIC.DB This table contains the mechanical information for each EIN as applicable.

\section{EIN_Code}

Size \& Type: A12*

Required: True

Folder:

Example: EIN000000033

Restrictions:

Information: This record identifier will be generated automatically by the system. It is a counter used to grant a unique ID for each EIN record.

Bearings_Sealed_F

Size \& Type: A15

Required:

Folder: Nameplate

Example: $\quad \mathrm{Y}$

Restrictions: $\mathrm{Y}$ or $\mathrm{N}$

Information: Front Bearing Sealed. This field is a boolean value represented by either a Y (Yes) or N (No).

\section{Bearings_Sealed_R}

Size \& Type: A15

Required:

Folder: Nameplate

Example: $\quad Y$

Restrictions: $\mathrm{Y}$ or $\mathrm{N}$

Information: Rear Bearing Sealed. This field is a boolean value represented by either a $Y(Y e s)$ or $N(N o)$.

Bearings_Vndr_No_F

Size \& Type: $\bar{A} 20$

Required:

Folder: Nameplate

Example:

Restrictions:

Information: Front Bearing Number.

Bearings_Vndr_No_R

Size \& Type: $\bar{A} 20$

Required:

Folder: Nameplate

Example:

Restrictions:

Information: Rear Bearing Number. 
LockID

Size \& Type: I

Required:

Folder: 1

Example:

Restrictions:

Information: This is the identifier for the lock session. This data is used internally by the system.

LockRec

Size \& Type: I

Required:

Folder:

Example: 1

Restrictions:

Information: This is the identifier for the record being modified. This data is used internally by the system.

RecordID

Size \& Type: +

Required:

Folder:

Example: 1

Restrictions:

Information: This is a record identifier used internally by the system.

PMS.DB This table contains 1 to many preventative maintenance records for each EIN as applicable.

EIN_Code

Size \& Type: A12*

Required: True

Folder:

Example: EIN000000033

Restrictions:

Information: This record identifier will be generated automatically by the system. It is a counter used to grant a unique ID for each EIN record.

PMS No

Size \& Type: A8*

Required: True

Folder: Pointer

Example: $\quad 9 \mathrm{X}-00003$

Restrictions: aa-nnunn

Information: Unique number JCS assigns to a specific job or activity. 
HNF-SD-WM-SDD-065

Revision 0

Page 32

LockID

Size \& Type: I

Required:

Folder:

Example:

Restrictions:

Information: This is the identifier for the lock session. This data is used internally by the system.

LockRec

Size \& Type: I

Required:

Folder:

Example:

Restrictions:

Information: This is the identifier for the record being modified. This data is used internally by the system.

RecordID

Size \& Type: +

Required:

Folder:

Example:

Restrictions:

Information: This is a record identifier used internally by the system.

PROCED.DB This table contains 1 to many procedure records for each EIN as applicable.

EIN Code

Size \& Type: A12*

Required: True

Folder:

Example: EIN000000033

Restrictions:

Information: This record identifier will be generated automatically by the system. It is a counter used to grant a unique $\mathrm{D}$ for each EIN record.

Procedure No

Size \& Type: A12*

Required: True

Folder: References

Example: $\quad 6-\mathrm{TF}-058-2 \mathrm{E}$

Restrictions:

Information: Procedure Number. Unique identifier for procedures, operating procedures, maintenance procedures,

Test procedures, etc. 
HNF-SD-WM-SDD-065

Revision 0

Page 33

Lockm

Size \& Type: I

Required:

Folder:

Example:

Restrictions:

Information: This is the identifier for the lock session. This data is used internally by the system.

LockRec

Size \& Type: I

Required:

Folder:

Example:

Restrictions:

Information: This is the identifier for the record being modified. This data is used internally by the system.

RecordID

Size \& Type: +

Required:

Folder:

Example:

Restrictions:

Information: This is a record identifier used internally by the system.

PROJECT.DB This table contains 1 to many project records for each EIN as applicable.

EIN Code

Size \& Type: A12*

Required: True

Folder:

Example: EIN000000033

Restrictions:

Information: This record identifier will be generated automatically by the system. It is a counter used to grant a unique ID for each EIN record.

Project No

Size \& Type: A10*

Required: True

Folder: $\quad$ Pointer

Example: B-534A

Restrictions:

Information: Unique identifier assigned to every Hanford Project. 
HNF-SD-WM-SDD-065

Revision 0

Page 34

LockID

Size \& Type: I

Required:

Folder:

Example:

Restrictions:

Information: This is the identifier for the lock session. This data is used internally by the system

LockRec

Size \& Type: I

Required:

Folder:

Example:

Restrictions:

Information: This is the identifier for the record being modified. This data is used internally by the system.

RecordID

Size \& Type: +

Required:

Folder:

Example:

Restrictions:

Information: This is a record identifier used internally by the system.

SYS_DWG.DB This table contains 1 to many system drawing records for each EIN as applicable.

EIN_Code

Size \& Type: A12*

Required: True

Folder:

Example: EIN000000033

Restrictions:

Information: This record identifier will be generated automatically by the system. It is a counter used to grant a unique ID for each EIN record.

Drawing_No

Size \& Type: All*

Required: True

Folder: References

Example: $\quad H-14-011733$

Restrictions: H-14-

Information: System Drawing Number. Unique number assigned to the H-14 O\&M system drawing. 
Sheets

Size \& Type: A3*

Required: True

Folder: References

Example: $\quad 001$

Restrictions $\mathrm{nnn}$

Information: System Drawing Sheet Number. Unique number assigned to the different sheets of the same System Drawing Number.

Revs

Size \& Type: A3

Required: True

Folder: References

Example: $\quad 001$

Restrictions: nnn

Information: System Drawing Revision Number. A unique number used to track the revision of a System Drawing.

Zones

Size \& Type: A2

Required:

Folder: References

Example: A1

Restrictions: An-Hn

Information: System Drawing Zone Location. Zone area where the EIN appears on the System Drawing.

XYZ_Coordinates

Size \& Type: A20

Required:

Folder: References

Example: $\quad 26.97521 .39800 .000$

Restrictions:

Information: System Drawing XYZ Coordinate Location. AutoCAD XYZ. Coordinates where the EIN appears on the System Drawing.

Drawing_Status

Size \& Type: A3

Required:

Folder:

Example:

Restrictions: lookup

Information: This is a link to the Dwgstat.DB, Status Description field. 
LockID

Size \& Type: I

Required:

Folder:

Example:

Restrictions:

Information: This is the identifier for the lock session. This data is used internally by the system.

LockRec

Size \& Type: I

Required:

Folder:

Example:

Restrictions:

Information: This is the identifier for the record being modified. This data is used internally by the system.

RecordID

Size \& Type: +

Required:

Folder:

Example:

Restrictions:

Information: This is a record identifier used internally by the system.

REF_DWG.DB This table contains 1 to many reference drawing records for each EIN as applicable.

EIN_Code

Size \& Type: A12*

Required: True

Folder:

Example: EIN000000033

Restrictions:

Information: This record identifier will be generated automatically by the system. It is a counter used to grant a unique $\mathrm{ID}$ for each ENN record.

Old_ID

Size \& Type: A20*

Required:

Folder: References

Example: WFT-03C-2

Restrictions:

Information: Alias or old identification number. 
HNF-SD-WM-SDD-065

Revision 0

Page 37

Reference_Source

Size \& Type: A20*

Required: True

Folder: References

Example: H-14-010112

Restrictions: H-14-

Information: System Drawing Number. Unique number assigned to the H-14 O\&M System Drawing.

Sheetr

Size \& Type: A3

Required: True

Folder: References

Example: $\quad 001$

Restrictions: nnn

Information: Reference Drawing Sheet Number. Unique number assigned to the different sheets of the same Reference Drawing Number.

Revr

Size \& Type: A3

Required: True

Folder: References

Example: 001

Restrictions: $\mathrm{nnn}$

Information: Reference Drawing Revision Number. A unique number used to track the revision of a System Drawing.

Zoner

Size \& Type: A2

Required:

Folder: References

Example: A1

Restrictions: An-Hn

Information: Reference Drawing Zone Location. Zone area where the EIN appears on the Reference Drawing.

$\mathrm{XYZ}$ Coordinater

Size \& Type: A20

Required:

Folder: References

Example: $\quad 26.97521 .39800 .000$

Restrictions:

Information: Reference Drawing XYZ Coordinate Location. AutoCAD XYZ. Coordinates where the EIN appears on the Reference Drawing. 
LockID

Size \& Type: I

Required:

Folder:

Example:

Restrictions:

Information: This is the identifier for the lock session. This data is used internaliy by the system.

LockRec

Size \& Type: I

Required:

Folder:

Example:

Restrictions:

Information: This is the identifier for the record being modified. This data is used internally by the system.

RecordID

Size \& Type: +

Required:

Folder:

Example:

Restrictions:

Information: This is a record identifier used internally by the system.

SAFE_SRC.DB This table contains 1 to many safety source document records for each EIN as applicable.

EIN_Code

Size \& Type: A12*

Required: True

Folder:

Example: EINO00000033

Restrictions:

Information: This record identifier will be generated automatically by the system. It is a counter used to grant a unique $\mathrm{ID}$ for each EIN record.

Source_Doc

Size \& Type: A25*

Required: True

Folder: Safety

Example:

Restrictions:

Information: Safety Source Document. Source documents are used to determine Safety Class. 
HNF-SD-WM-SDD-065

Revision 0

Page 39

Source_Sec

Size \& Type: A10*

Required: True

Folder: Safety

Example:

Restrictions:

Information: Safety Source Document Section. Source Document Section used to determine Safety Class.

LockID

Size \& Type: 1

Required:

Folder:

Example:

Restrictions:

Information: This is the identifier for the lock session. This data is used internally by the system.

LockRec

Size \& Type: I

Required:

Folder:

Example:

Restrictions:

Information: This is the identifier for the record being modified. This data is used internally by the system.

RecordID

Size \& Type: +

Required:

Folder:

Example:

Restrictions:

Information: This is a record identifier used internally by the system.

SAFE_TNK.DB This table contains 1 to many tank records for each EIN as applicable.

EIN_Code

Size \& Type: A12*

Required: ' True

Folder:

Example: EIN000000033

Restrictions:

Information: This record identifier will be generated automatically by the system. It is a counter used to grant a unique ID for each EIN record. 
Tank_ID

Size \& Type: A10*

Required: True

Folder: Safety

Example:

Restrictions:

Information: Tank Identification. Associated tanks.

LockID

Size \& Type: I

Required:

Folder:

Example:

Restrictions:

Information: This is the identifier for the lock session. This data is used internally by the system.

LockRec

Size \& Type: I

Required:

Folder:

Example:

Restrictions:

Information: This is the identifier for the record being modified. This data is used internally by the system.

RecordID

Size \& Type: +

Required:

Folder:

Example:

Restrictions:

Information: This is a record identifier used internally by the system.

SPR PRTS.DB This table contains 1 to many spare parts records for each EIN as applicable.

\section{EIN Code}

Size \& Type: A12*

Required: True

Folder:

Example: EIN000000033

Restrictions:

Information: This record identifier will be generated automatically by the system. It is a counter used to grant a unique ID for each EIN record. 
Sp_Parts_No

Size \& Type: A10*

Required: True

Folder: Pointer

Example: $6167-1411-11$

Restrictions nnnn-mnn-

Information: Spare Part Number. Unique identifier used as the key field in the Spare Parts system.

LockID

Size \& Type: I

Required:

Folder:

Example:

Restrictions:

Information: This is the identifier for the lock session. This data is used internally by the system.

LockRec

Size \& Type: I

Required:

Folder:

Example:

Restrictions:

Information: This is the identifier for the record being modified. This data is used internally by the system.

RecordID

Size \& Type: +

Required:

Folder:

Example:

Restrictions:

Information: This is a record identifier used internally by the system.

ECN.DB This table contains 1 to many procedure records for each EIN as applicable.

EIN Code

Size \& Type: A12*

Required: True

Folder:

Example: EIN000000033

Restrictions:

Information: This record identifier will be generated automatically by the system. It is a counter used to grant a unique ID for each EIN record. 
HNF-SD-WM-SDD-065

Revision 0

Page 42

ECN

Size \& Type: A10*

Required: True

Folder: References

Example: 608038

Restrictions:

Information: ECN Number. Unique identifier for ECNs.

LockD

Size \& Type: I

Required:

Folder:

Example:

Restrictions:

Information: This is the identifier for the lock session. This data is used internally by the system.

LockRec

Size \& Type: I

Required:

Folder:

Example:

Restrictions:

Information: This is the identifier for the record being modified. This data is used internally by the system

RecordID

Size \& Type: +

Required:

Folder:

Example:

Restrictions:

Information: This is a record identifier used internally by the system.

SYSTEMS.DB This table contains the lookup values for the various systems.

System

Size \& Type: A5*

Required: True

Folder: Utility / Definitions / EIN Systems

Example: CHMA

Restrictions:

Information: This is the unique link field for the system value. It is an abbreviation of the system name. 
HNF-SD-WM-SDD-065

Revision 0

Page 43

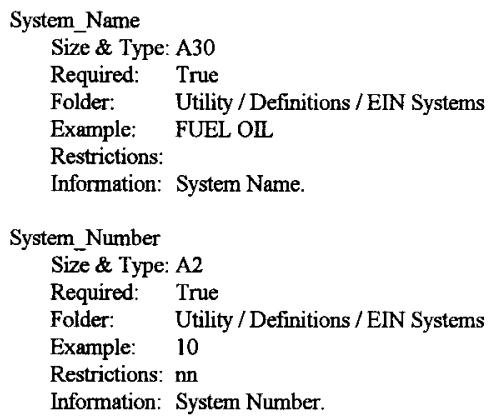

COMP_DES.DB This table contains the lookup values for the various components.

Component

Size \& Type: A5*

Required: True

Folder: Utility / Definitions / EIN Components

Example: AOD

Restrictions:

Information: This is the unique link field for the component value. It is an abbreviation of the component name.

Comp_Name

Size \& Type: A55

Required: True

Folder: Utility / Definitions / EIN Components

Example: BATTERY

Restrictions:

Information: Component Name.

FAC_FARM.DB This table contains the lookup values for the various farm facilities.

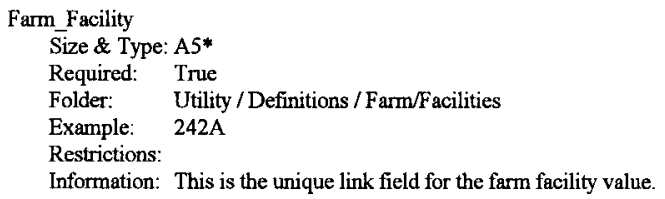


HNF-SD-WM-SDD-065

Revision 0

Page 44

Structure_Description

Size \& Type: A65

Required: True

Folder: Utility / Definitions / Farm/Facilities

Example: This is the AN Farm.

Restrictions:

Information: Description of the structure.

EINSTAT.DB This table contains the lookup values for the various EIN statuses.

Status

Size \& Type: A3*

Required: True

Folder: Utility / Definitions / EIN Statuses

Example: 1

Restrictions:

Information: This is the unique link field for the status value.

Status_Description

Size \& Type: $\mathrm{A} 30$

Required: True

Folder: Utility / Definitions / ENN Statuses

Example: Label Made

Restrictions:

Information: Status Description

Default_Initial_Status

Size \& Type: L

Required: True

Folder: Utility / Definitions / EN Statuses

Example:

Restrictions:

Information: Default Initial Status, boolean value.

NOUN_ABB.DB This table contains the lookup values for the various noun names.

Noun_Name_Abbreviation

Size \& Type: Al0*

Required: True

Folder: Utility / Definitions / Noun Name Abbreviations

Example: ADJ

Restrictions:

Information: This is the unique link field for the noun name value. 
HNF-SD-WM-SDD-065

Revision 0

Page 45

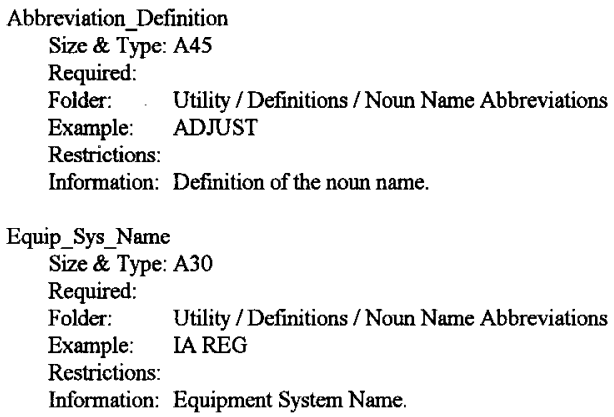

COMP_TYP.DB This table contains the lookup values for the various component types.

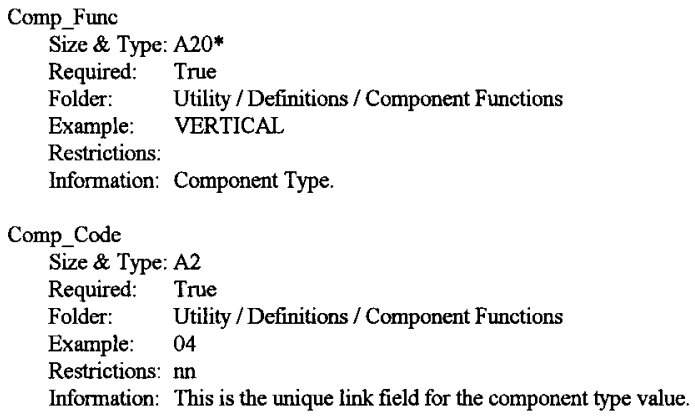

DWGSTAT.DB This table contains the lookup values for the various drawing statuses.

\section{Status}

Size \& Type: A3*

Required: True

Folder: Utility / Definitions / Drawing Statuses

Example: 1

Restrictions:

Information: This is the unique link field for the status value. 
StatusDescription

Size \& Type: A30*

Required: True

Folder: Utility / Definitions / Drawing Statuses

Example: Label Made

Restrictions:

Information: Status Description

Default_Initial_Status

Size \& Type: L

Required: True

Folder: Utility / Definitions / Drawing Statuses

Example:

Restrictions:

Information: Default Initial Status, boolean value

USER.DB This table contains the MEL users and related information.

Hanford_No

Size \& Type: A12*

Required: True

Folder: Admin / Access Control

Example: H0068719

Restrictions: Hnnnnnnn

Information: Hanford ID. Unique link field.

Name

Size \& Type: A40

Required: True

Folder: Admin / Access Control

Example: Doe, J.D.

Restrictions:

Information: User name.

Privilege

Size \& Type: A8

Required: True

Folder: Admin / Access Control

Example: $\quad 11111111$

Restrictions: nnnnnm

Information: User privilege. Automatically generated by the system. 
Password

Size \& Type: Alo

Required:

Folder: Admin / Access Control

Example: TESTER

Restrictions:

Information: User password.

REASONS.DB This table contains the values for the various reasons for data editing.

\section{Reasons}

Size \& Type: A30

Required: True

Folder: Utilities / Definitions / Edit Reasons

Example: Delete Record

Restrictions:

Information: Reason description.

\subsubsection{SYSTEM TABLES}

EIN_IN.DB This table contains the next available EIN_Code.

EIN Code

Size \& Type: A12*

Information: This record identifier will be generated automatically by the system. It is a counter used to grant a unique ID for each EIN record.

LockID

Size \& Type: I

Required:

Folder:

Example:

Restrictions:

Information: This is the identifier for the lock session. This data is used internally by the system.

SessionNo

Size \& Type: I

Required:

Folder:

Example:

Restrictions:

Information: This is the identifier for the session. This data is used internally by the system. 
RPT_SQLS.DB This table contains the SQL queries.

\section{Table Name}

Size \& Type: A8*

Information: This value specifies which table the query is specified for.

SQL_Query

Size \& Type: M240

Information: This field contains the SQL code for the query associated with the appropriate table.

\section{SESSNLOG.DB This table logs data relevant to the edit session.}

\section{SessionID}

Size \& Type: $\mathrm{I}^{*}$

Information: This record identifier will be generated automatically by the system. It uniquely identifies a session.

SessionReleaseNo

Size \& Type: I*

Information:

\section{SessionUser}

Size \& Type: $\mathrm{A} 40$

Information: User name for the edit session.

SessionDate

Size \& Type: A20

Information: Date for the edit session.

SessionTime

Size \& Type: A20

Information: Time for the edit session.

SessionReason

Size \& Type: A30

Information: Reason for the edit session.

\section{SessionDocument}

Size \& Type: A25

Information: Document related to the edit session.

ReleaseUser

Size \& Type: A40

Information: User name of release verification individual.

ReleaseDate

Size \& Type: A20

Information: Date of release. 
HNF-SD-WM-SDD-065

Revision 0

Page 49

ReleaseTime

Size \& Type: A20

Information: Time of release.

LOCKS.DB This table logs lock user information relevant to set of locked data records.

LockID

Size \& Type: +*

Information: Automatically incremented unique lock identifier.

LockUser

Size \& Type: A40

Information User name of individual locking data for modification.

LockDate

Size \& Type: A20

Information: Date of lock session.

LockTime

Size \& Type: A20

Information: Time of lock session.

LockReason

Size \& Type: A30

Information: Reason for locking data.

LockDocument

Size \& Type: A25

Information: Document related to lock session.

CHANGLOG.DB This table logs all data modifications.

SessionID

Size \& Type: $I^{*}$

Information: Identifer for change session.

SessionReleaseNo

Size \& Type: I*

Information: Identifier for the release number.

SessionRec

Size \& Type: I*

Information: Identifier for changed record. 
EIN

Size \& Type: A23*

Information: Modified EIN.

ChangedTable

Size \& Type: A12*

Information: Name of table containing change.

ChangedField

Size \& Type: A25*

Information: Name of field containing change.

Action

Size \& Type: A6

Information: Type of change.

NewValue

Size \& Type: A30

Information: New value.

OldValue

Size \& Type: A30

Information: Old value.

LOCKDATA.DB This table logs the data relevant to a lock session.

LockID

Size \& Type: I*

Information: Link field to the Lock information in Locks.DB.

LockRec

Size \& Type: $I^{*}$

Information: Identifier for the locked record.

EIN

Size \& Type: A23*

Information: Locked EIN.

LockedTable

Size \& Type: A12*

Information: Locked Table.

LockedField

Size \& Type: A25*

Information: Locked Field. 
HNF-SD-WM-SDD-065

Revision 0

Page 51

Action

Size \& Type: $A 6$

Information: Reason for locking data.

PendingValue

Size \& Type: A60

Information: New data value pending verification.

CurrentValue

Size \& Type: A60

Information: Data value before lock.

QRYFLDS.DB This table contains field information associated with a query.

Order

Size \& Type: $I^{*}$

Information: Order of table records.

TableName

Size \& Type: A12

Information: Name of table.

FieldName

Size \& Type: A25

Information: Name of field.

DataGroup

Size \& Type: A15

Information: Name of data group.

DataType

Size \& Type: A1

Information: Type of data.

LookupTableName

Size \& Type: A12

Information: Name of lookup table.

LookupFieldName

Size \& Type: A25

Information: Name of lookup field.

DisplayName

Size \& Type: A25

Information: User-friendly display name. 
HNF-SD-WM-SDD-065

Revision 0

Page 52

\section{EditMask}

Size \& Type: A75

Information: System compatible field mask identifying required and optional character specifications.

\section{DisplayMask}

Size \& Type: A60

Information: User-friendly version of the edit mask used for user help. 
HNF-SD-WM-SDD-065

Revision 0

Page 53

\subsection{PROGRAMMUNG}

\subsection{PLATFORM}

The MEL Phase I will operate on the user's desktop PC under the current Hanford End User Operating Environment (ESOE). Currently, this includes Windows Version 3.11 (Windows for Workgroups) and DOS Version 6.22 and Windows95. Users that do not operate under this configuration will be unable to execute the MEL system.

All MEL data will be stored in Borland Paradox for Windows Version 5.0 data tables. These are binary files that may only be read using a database table interpreter or "engine".

Borland Delphi Version 1.0 will be used as the application development tool for the MEL system. Delphi is a fourth generation object oriented programming language (4GL) that provides the developer with the flexibility needed to satisfy all the MEL requirements in the most efficient and cost-effective manner.

Several third-party component packages will be used in building the MEL system. A component is a single binary file that may be directly integrated into the Borland Delphi development environment. Although these components are transparent to the common user, they provide the developer with enhanced programming capabilities. Components used in the MEL include InfoPower Version 1.0 and Crystal Reports Component Version 7.0. Infopower is a package of components which provide the developer with enhanced data aware modules, while Crystal Reports Component is a single component which allows the integration of custom reports.

Installation of the MEL will be accomplished using the OnDemand WINStall Version 5.0 program. This application has the flexibility to be configured specifically for the needs of the MEL system. The WINStall program files specific to MEL will be maintained on the MEL fileserver.

Crystal Reports Version 4.5 by Seagate Inc. will be used for the development of the MEL reports. Crystal Reports is a separate program that is flexible enough to support multiple types of databases and programming languages. These reports will be integrated into the MEL system using the Crystal Reports Component discussed above.

The MEL database tables will be stored on a fileserver using Windows NT Version 3.51 as the operating system. This configuration allows the fileserver administrator to setup "user groups" which are groups of individuals allowed access the MEL data. 
HNF-SD-WM-SDD-065

Revision 0

Page 54

\subsection{USER INTERFACE}

\subsubsection{PROGRAM UNITS}

The MEL system, written in Borland Delphi 1.0, is compiled into a single executable file which is placed on the users desktop PC during the MEL installation process. However, the single executable file is actually made up of several Pascal files (Pascal files end with a .pas extension). Pascal files contain the actual source code and are native to Borland Delphi, since Pascal was used in the development of Delphi.

Pascal files (which are referred to as "units" in Delphi) will be strategically designed for the MEL system. A description of each is as follows:

ABOUT.PAS - This unit contains the source code for the "About" dialog screen.

ADDEIN.PAS - This unit contains the source code for the "Add New EINs" dialog screen.

CHG_ACTV.PAS - This unit contains the source code for the "Change Control Activity" dialog screen.

CHGPNDG.PAS - This unit contains the source code for the "Changes Pending" dialog screen.

EDITVIEW.PAS - This unit contains the source code for the editview (folders) screen.

FIND.PAS - This unit contains the source code for the find dialog screen.

INSPNDG.PAS - This unit contains the source code foe the new records dialog screen.

JUSTIFY.PAS - This unit contains the source code for the edit reason dialog screen.

LOGON.PAS - This unit contains the source code for the "Logon" dialog screen.

MAIN.PAS - This unit contains the source code for the MEL Phase I main screen. This includes the navigation tools, main menu and control buttons.

PRNTFILE.PAS - This unit contains the source code for the "Export File Type" dialog screen. 
HNF-SD-WM-SDD-065

Revision 0

Page 55

PRNTLOCK.PAS - This unit contains the source code for the "Print Data Locks" dialog screen.

QRY_RES.PAS - This unit contains the source code for the "Query Results" dialog screen.

QRYWAIT.PAS - This unit contains the source code for the "Preparing your report, please wait" dialog screen.

QUERY.PAS - This unit contains the source code for the "Query" dialog screen.

REPORTS.PAS - This unit contains the source code for the "Reports" dialog screen.

SPLASH.PAS - This unit contains the source code for the MEL splash screen, which is visible at startup.

STDDEFS.PAS - This unit contains the source code for the "Standard Data Definitions" dialog screen.

USERS.PAS - This unit contains the source code for the "User Information" dialog screen.

\subsubsection{EDITVIEW UNIT}

The complexity of the Editview unit requires that it be documented in explicit detail. Other units in the MEL are not as complex, and are therefore self-documenting.

Below is a list of the procedures and functions in the frmEditView unit for the MEL, phase I, version 2.0. The list contains routine declarations as they appear (and in the same order they appear) in the unit's source file, EDITVIEW.PAS. Where useful, a brief description of the routine's function is provided. Also, they are cross-referenced to the MEL, Phase I Data Editing Structure Charts. One or more numbers in square brackets, e.g., [2, 4], indicate the structure chart pages that illustrate the function or procedure.

\section{Version 1.0 routines kept...}

procedure FormCreate (Sender: TObject);

procedure SetPointerFolder (mode: String);

procedure SetReferenceFolder (mode: String);

procedure SetNameplateFolder (mode: String); 
HNF-SD-WM-SDD-065

Revision 0

Page 56

procedure SetSpecificationsFolder (mode: String);

procedure SetSafetyFolder (mode: String);

procedure

SetLabelAdminAccess (mode: String);

procedure

MEL_TabsChange (Sender: TObject; NewTab: Integer; var AllowChange: Boolean);

Data entry format checking (where 'masks' leave off)...

procedure fldSafetyClassChange (Sender: TField);

procedure fldOSRChange (Sender: TField);

procedure flenvironmentalChange (Sender: TField);

procedure fldQAChange (Sender: TField);

procedure fldBearingsSealedFChange (Sender: TField);

procedure ndBearingsSealedRChange (Sender: TField);

procedure fldZonerChange (Sender: TField);

procedure

function

fldSysZonesChange (Sender: TField);

procedure

function

CheckZoneValue ( zone: String ):Boolean;

ForceUpperCase (Sender: TField);

procedure

procedure

AutoFillLead ( s: string; sz: integer; c: string ): string;

fldSysRevsChange (Sender: TField);

procedure

fldSysSheetsChange (Sender: TField);

procedure

fidRefRevrChange (Sender: TField);

fldRefSheetrChange (Sender: TField);

Event handlers to capture changes...

procedure dsUpdateData (Sender: Tobject);

This handler is fired (by the Post or UpdateRecord methods of a dataset) when the current record is about to be updated in the database. This event occurs early enough that an actual post can be canceled.

procedure $\quad$ grdKeyDown (Sender: TObject; var Key: Word;

Shift: TshiftState);

Capture attempts to insert and delete records. These operations must be performed via the pop up menu.

procedure System_LU_boxEnter (Sender: TObject);

procedure Comp_LU_boxEnter (Sender: TObject); 
HNF-SD-WM-SDD-065

Revision 0

Page 57

procedure Status_LU_boxEnter (Sender: TObject);

procedure Fac_LU__boxEnter (Sender: Tobject);

procedure System_LU_boxCloseUp (Sender: TObject; LookupTable: TwwTable; FillTable: TDataset; modified: Boolean);

procedure Comp_LU_boxCloseUp (Sender: TObject; LookupTable: TwwTable; FillTable: TDataset; modified: Boolean);

procedure Status_LU_boxCloseUp (Sender: TObject; LookupTable: TwwTable; FillTable: TDataset; modified: Boolean);

procedure Fac_LU_boxCloseUp (Sender: TObject; LookupTable: TwwTable; FillTable: TDataset; modified: Boolean);

procedure EvaluateEIN;

Pop up menu invocation and function setup support...

The pop up menu of options for data fields is always accessible to the user when a right button mouse click is made on an edit box or grid. Upon right click, the following processes are invoked.

procedure edMouseDown (Sender: TObject; Button: TmouseButton;

[1]

$$
\text { Shift: TShiftState; X, Y: Integer); }
$$

This handler is attached to all TDBEdit components used to edit MEL data fields. If the mouse click is on the right button, this save the associated table and data source and call invoke the pop up menu, popEditor. Although it would seem more direct to specify the pop up menu in each of the TDBEdit components (which is then automatically invoked by a rightclick on the edit box), this prevents the table and data source associated with the edit box from being identified.

procedure $\quad$ grdMouseDown (Sender: TObject; Button: TmouseButton;

[1]

$$
\text { Shift: TShiftState; X, Y: Integer); }
$$

This handler is attached to the TwwDBGrid components used to edit MEL data and serves the same purpose as the edMouseDown handler but for grids.

procedure popEditPopup (Sender: Tobject); 
HNF-SD-WM-SDD-065

Revision 0

Page 58

This handler is attached to the popEditor pop up menu component. When called, this procedure determines the current user session conditions and enables or disables the individual menu items appropriately.

After this handler executes, the pop up menu is displayed to the user. Processes are suspended until the user makes a menu selection or moves off the menu and activates another handler elsewhere (effectively canceling the menu).

When the pop up menu is displayed, the user may select an enabled menu item or click elsewhere on the display. Each menu item has it's own event handler named 'piXXXClick' where XXX is the name of the selected item.

\section{Pop up menu item handlers...}

procedure piChangesClick (Sender: Tobject);

[2]

The user has requested to view the changes pending for the selected table, record, and field. Make sure any edits in progress are cleaned up, get the pertinent information, then display the dialog for changes pending. If the user is the owner of the data, we're in edit mode, and the changes pending do not represent a deletion, allow editing within the dialog. Update displayed data accordingly upon return.

procedure piNewRecordsClick (Sender: Tobject);

[2]

The user has requested to view all new records (insertions) for the selected table. Display the dialog for new records pending.

procedure piLockClick (Sender: Tobject);

[3]

Lock the selected table \& record for the user. Do not enable for editing.

procedure piEditClick (Sender: Tobject);

[3]

The user wants to edit the selected table \& record. Lock the record for the user and enable editing. If the selected field is in the EIN_INFO table, all dependent records in the other tables must be free or owned by the editing user. 
HNF-SD-WM-SDD-065

Revision 0

Page 59

procedure piPostClick (Sender: Tobject);

[4]

The user wants to post any changes pending for the single selected record. Confirm post with user.

procedure piCancelClick (Sender: Tobject);

[6]

The user wants to cancel any changes pending for the single selected record. Confirm cancellation with user.

procedure piDeleteClick (Sender: Tobject);

[7]

The user wants to mark the selected record for deletion. Get confirmation, clean up edits, lock the record, and mark it for deletion.

procedure piNewClick (Sender: Tobject);

[8]

The user wants to insert a new record. Clean up any open edits. Initialize a new record for the table, lock it, enable editing, etc. Note - the record will later be deleted after field entries are recorded.

\section{Data operations access support...}

function IsPrivileged ( $\mathrm{pIdx}$ : integer ): Boolean;

[1]

Return true only if the session user has correct privilege to edit the folder indexed by the given index.

function IsOwner ( lockID: integer): Boolean;

[1]

Return true if the session user owns the selected record. 
function

$[1,2]$
GetPendingAction ( lockID, lockRec: integer;

ein, tName, fName: string ): string;

Return the action pending for the first LOCKDATA record matching the given parameters. If no matching record is located, return 'Error'.

function

AnyNewRecords ( ein, tName: string ): Boolean;

[1]

Determine if there are any new records pending (insertions) for the given EIN and table. If so, return true.

\section{Lock and change indication support...}

procedure DetermineFieldColors ( $\mathrm{tbl}$ : TwwTable; var fg, bg: TColor );

[2]

Determine the appropriate foreground (font) and background colors for the current record in the given table. This is the only function used to determine these colors.

procedure UpdateFieldColors;

$[2,9]$

Force an update of all field colors by triggering the OnChange handler for the tables.

procedure EINInfoLockIDChange (Sender: Tobject);

[2]

This handler fires whenever the LockID in the EIN_Info table changes as the result of navigating records or changing the current record's lock status. Every edit box associated with the table must be updated separately.

procedure EqpInfoLockIDChange (Sender: Tobject);

[2]

This handler fires whenever the LockID in the Eqp_Info table changes as the result of navigating records or changing the current record's lock status. Every edit box associated with the table must be updated separately. 
HNF-SD-WM-SDD-065

Revision 0

Page 61

procedure MotorLockIDChange (Sender: Tobject);

[2]

This handler fires whenever the LockID in the Motor table changes as the result of navigating records or changing the current record's lock status. Every edit box associated with the table must be updated separately.

procedure ElectricLockIDChange (Sender: Tobject);

[2]

This handler fires whenever the LockID in the Electric table changes as the result of navigating records or changing the current record's lock status. Every edit box associated with the table must be updated separately.

procedure MechanicLockIDChange (Sender: Tobject);

[2]

This handler fires whenever the LockID in the Electric table changes as the result of navigating records or changing the current record's lock status. Every edit box associated with the table must be updated separately.

procedure grdCalcCellColors (Sender: TObject; Field: TField; State:

TgridDrawState; highlight: Boolean; AFont: TFont; ABrush: Tbrush);

Set the font and background colors for each cell. Function DeterminFieldColors will determine the appropriate colors based upon the lockID for each record.

procedure ShowPreviousChanges ( tbl: TwwTable); $[2,3]$

Replace the viewed data with values from the changes pending. This display of changes is temporary and local.

\section{Lock and Edit support...}

function LockSingleRecord ( tbl: TwwTable; dsrc: TwwDataSource;

[3]

lockedit: integer; einCode: string ): Boolean; 
If available, lock the current record in the given table. Create a new lockID if needed. Return true if record is locked to vUser.

function LockManyRecords ( tbl: TwwTable; dsrc: TwwDataSource; [3] lockedit: integer; einCode: string ): Boolean; Lock all records in the given table that match the given einCode. Create a new LockID if necessary. Return true if successful, false if not.

function LockEINRecord ( einCode: string ): Boolean; $[3,7]$

Check all associated records for the EIN before locking. If any records are locked, return false. If not, lock them and return true.

function GetSingleLockStatus ( tbl: TwwTable; einCode: string ): integer; [3]

Return the status of the current single record in the given table. If the record's ein_code does not match, return $\mathrm{cNoRecord}$.

function GetManyLockStatus ( tbl: TwwTable; einCode: string ): integer; [3]

Return the combined status for all records in the given table, tbl, that match the given einCode.

function CreateLock ( user, reason, document: string ): integer; $[3,8]$

Create an entry in the LOCKS table and return the new lockID.

procedure CopyRecord ( tbl: TwwTable ); $[2,3,7,8]$

Copy the names of the selected table fields and then copy the contents of the fields into a memory array. 
HNF-SD-WM-SDD-065

Revision 0

Page 63

procedure

Detour ( dsrc: TwwDataSource );

$[3,4,6,9]$

This routine acts as a detour for data when a normal post of changes would occur. Record changes in the LOCKDATA table and cancel the impending post. If the record was just inserted, delete it from the database table.

procedure SaveDatabaseRecord ( tbl: TwwTable; action: string );

$[3,8]$

Save the current record for the given table, 'tbl', in LOCKDATA with the given 'action'. At this point, the tbl is already pointing to the record of interest, the current field values have been saved in the vRcdValues TSringList, and the field names have been copied into the vRcdFields TstringList.

procedure SaveDatabaseChanges ( tbl: TwwTable);

[9]

Save the values of changed fields for the current record of the given table, 'tbl', in LOCKDATA. At this point, the tbl is already pointing to the record of interest, the original field values have been saved in the vRcdValues TSringList, and the field names have been copied into the vRcdFields TstringList.

procedure SaveDatabaseDeletion ( tbl: TwwTable );

[7]

Save the values of fields for the current record of the given table, 'tbl', in LOCKDATA - they are to be deleted. At this point, the tbl is already pointing to the record of interest, the original field values have been saved in the vRcdValues TStringList, and the field names have been copied into the vRcdFields TstringList.

procedure SaveDatabaseDeletions ( tbl: TwwTable; einCode: string);

[7]

Save all records in the given table that match the current EIN.

procedure SaveEINDeletion ( einCode: string);

[7]

Save each of the constituent records for an EIN. 
procedure InitDatabaseRecord ( tbl: TwwTable);

[8]

Initialize the new record in the given table.

procedure CreateLockDataRecord ( lockID, lockRec: integer;

[8]

$$
\text { newValue, oldValue, action, ein, tblName, fldName: string ); }
$$

Insert a new record in the LOCKDATA table and fill with the given data.

procedure UpdateLockDataRecord (newValue, oldValue, action: string );

$[7,9]$

The correct LOCKDATA record is already pointed at by tblLockData. Change the pending value, current value, and action as given.

function LocateLockDataRecord (ein, tName, fName: string;

$[1,2,7,9]$

$$
\text { lockID, lockRec: integer ): Boolean; }
$$

Given the match parameters required, locate the LOCKDATA record and leave the tblLockData pointing at the record. Return true if the match is successful, false if not found.

\section{Cancel support...}

function CancelChangesPendingRecord (lockI, lockRec: integer; $[5,6]$

$$
\text { ein, tName: string ): Boolean; }
$$

Remove the lock on the given table and record and remove the associated LOCKDATA entries. Remove the LOCKS entry if appropriate. Return true if successful, false if not.

function CancelEINChangesPending ( lockID: integer; ein: string ): Boolean; [6]

Remove the locks on all records in all database tables for the given EIN. Then remove the associated LOCKDATA and the LOCKS entry if appropriate. Return true if successful, false if not. 
HNF-SD-WM-SDD-065

Revision 0

Page 65

function CancelSessionChangesPending ( lockID: integer ): Boolean;

Cancel all locks and changes made in one session by a specific user. Return true if successful, false if not.

function CancelUsersChangesPending (user: string ): Boolean;

Cancel all locks and changes made in any sessions by a specific user. Return true if successful, false if not.

function CancelAllChangesPending: Boolean;

Cancel all locks and changes pending. Return true if successful, false if not.

function UnlockDatabaseRecord ( lockID, lockRec: integer;

[6]

einCode, tName: string ): Boolean;

Remove the LockID and LockRec from a single data table record. Return true if successful, false if not.

function UnlockEINDatabaseRecords ( einCode: string ): Boolean;

[6]

Remove the LockID (and LockRec) from ALL data tables records with the matching einCode. Return true if successful, false if not.

function UnlockSessionDatabaseRecords ( lockID: integer ): Boolean;

Remove the LockID (and LockRec) from the data tables records with the given lockID.

Return true if successful, false if not.

function UnlockAllDatabaseRecords: Boolean;

Remove the LockID (and LockRec) from ALL data tables records. Return true if successful, false if not.

function DeleteLockDataLockRec (lockID, lockRec: integer;

$[5,6]$

ein, tName: string ): Boolean; 
Remove all LockData entries for the given LockID and LockRec. Return true if successful, false if not.

function DeleteEINLockData ( lockID: integer; ein: string ): Boolean; [6]

Remove all LOCKDATA entries for the given LockID and EIN. Return true if successful, false if not.

function DeleteSessionLockData ( lockID: integer ): Boolean;

Return true if successful, false if not.

function DeleteAllLockData: Boolean;

Return true if successful, false if not.

function CleanupLocks ( LockID: integer): Boolean;

$[5,6]$

If no LockData entries exist for the LockID, clear the associated Locks entry. Return true if successful, false if not.

function ClearLocks: Boolean;

Clear the Locks. db table. Return true if successful, false if not.

Post support...

function PostChangesPendingRecord (lockID, lockRec: integer;

$[4,5]$

$$
\text { ein, tName: string ): Boolean; }
$$

Post the pending changes to a specific data table \& record and release. Return true if successful, false if not.

function PostEINChangesPending (lockID: integer; ein: string ): Boolean;

Post all changes made for the given EIN. Return true if successful, false if not. 
HNF-SD-WM-SDD-065

Revision 0

Page 67

function

PostSessionChangesPending ( lockID: integer ): Boolean;

Post all the changes made in one session. Return true if successful, false if not.

function PostUsersChangesPending (user: string ): Boolean;

Post all changes made by a given user. Return true if successful, false if not.

function PostAllChangesPending: Boolean;

Post all changes pending. Return true if successful, false if not.

function RecordIsLockedOnly ( lockID, lockRec: integer;

[4]

ein, tName: string ): Boolean;

If all matching LOCKDATA records have "Lock' action, return true.

function LogPostingSession ( lockID: integer): integer;

[4]

Create a session log record based upon the locks record and the current user making the release. Return the SessionID.

function LogAction ( lockID, releaseNo, lockRec: integer;

[5]

$$
\text { ein, tName: string ): Boolean; }
$$

Copy the LOCKDATA records to the CHANGLOG for the given record set. Return true if successful, false if not.

function GetSessionReleaseNo (lockID: integer): integer;

[4]

Search SESSNLOG for lockID. If found get largest associated SessionReleaseNo, increment it and return it. If lockID not found, return 1.

function GetFirstAction ( lockID, lockRec: integer;

[4]

ein, tName: string ): string; 
HNF-SD-WM-SDD-065

Revision 0

Page 68

For a given lockID and lockRec, get the action for the first matching LOCKDATA record.

function DeleteDatabaseRecord ( lockID, lockRec: integer;

[5]

$$
\text { einCode, tName: string ): Boolean; }
$$

If the table is not EIN_Info, delete the single record. If it is the EIN_Info table, delete the record and all associated records in all of the other data tables (matching EIN_Codes).

Return true if successful, false if not.

function InsertDatabaseRecord ( lockID, lockRec: integer;

[5]

$$
\text { ein, tName: string ): Boolean; }
$$

If the table is not EIN_Info, insert a new record in the table and fill it with the values in LOCKDATA. If the table is EIN_Info, a new EIN_Code must be created. Return true if successful, false if not.

function UpdateDatabaseRecord ( lockID, lockRec: integer;

[5]

$$
\text { ein, tName: string ): Boolean; }
$$

Modify the fields in the single table/record which have 'Change' action LOCKDATA records. Leave 'Lock'ed fields unchanged. Return true if successful, false if not.

\section{General support...}

procedure EnableTable (dsrc: TwwDataSource);

$[2,3,8]$

Turn on the auto-edit property for the data source.

procedure DisableTable ( dsrc: TwwDataSource);

$[3,9]$

Turn off the auto-edit property for the data source.

procedure CheckForEdits;

$[3,4,6,7,8]$ 
If a table is open for editing, call Detour to record and clean up.

function

GetNewEINCode: String;

$[5,8]$

Open the EIN_No.DB table to obtain the next available number to be applied to an EIN_Code. Build the new EIN_Code value. If successful, post the new value back to the EIN_No.DB.

function OkTOAddRecord ( tbl: TwwTable ): Boolean;

[8]

Determine if a new record is appropriate for the given table. This function does not consider the contents of LOCKDATA. Return true if Ok.

function GetOwner ( lockID: integer ): string;

$[1,2,3]$

Return the name of the user that owns the LockID.

function GetEINCode (ein: string ): string;

$[4,5,6]$

Retrieve the EIN_Code for a given EIN. Don't change the EINInfo table position but check it first to see if the EIN matches.

procedure RefreshAll;

$[4,6,7]$

function Stripper ( textin: string ): string; 


\subsubsection{EDITVIEW STRUCTURE CHARTS}

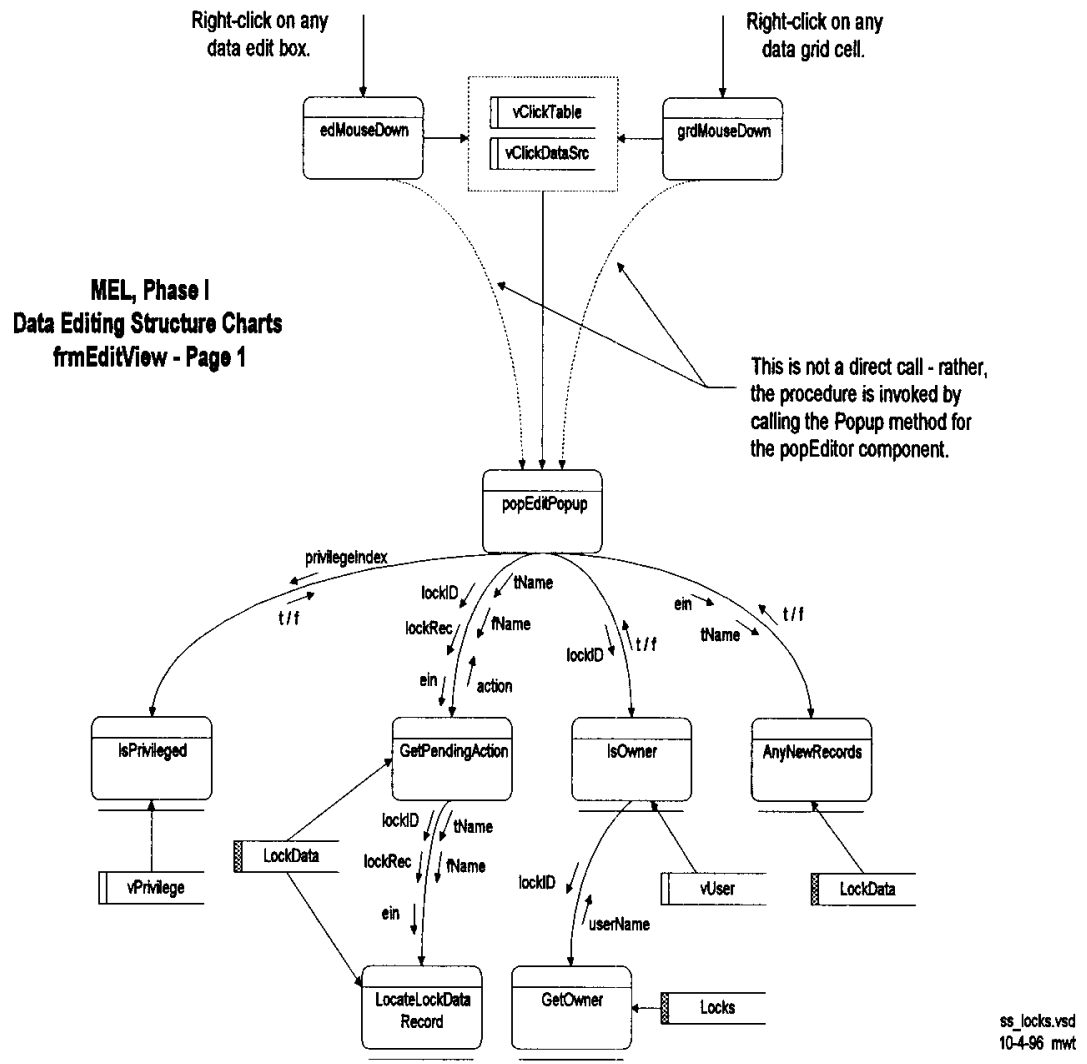


HNF-SD-WM-SDD-065

Revision 0

Page 71

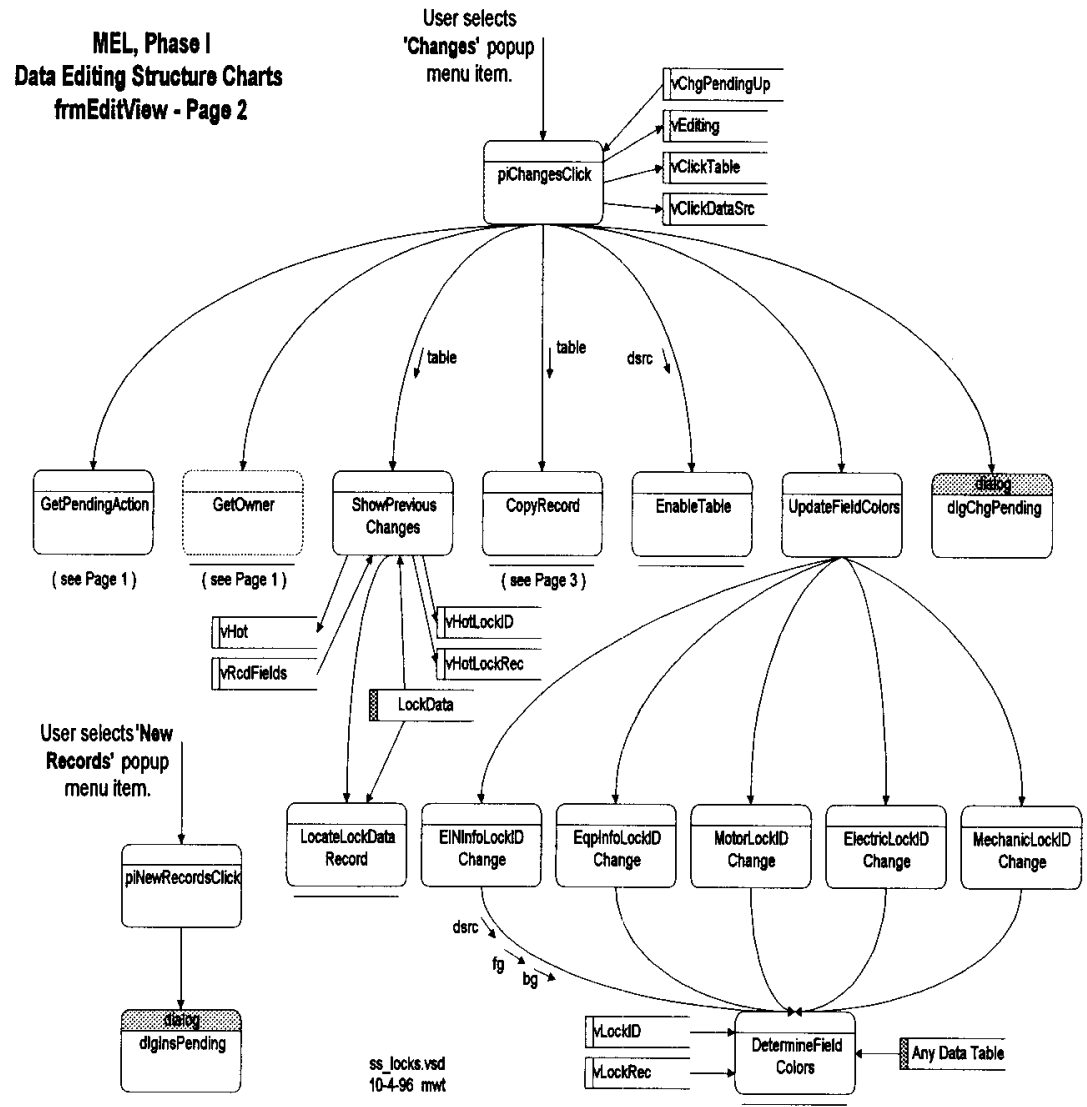


HNF-SD-WM-SDD-065

Revision 0

Page 72

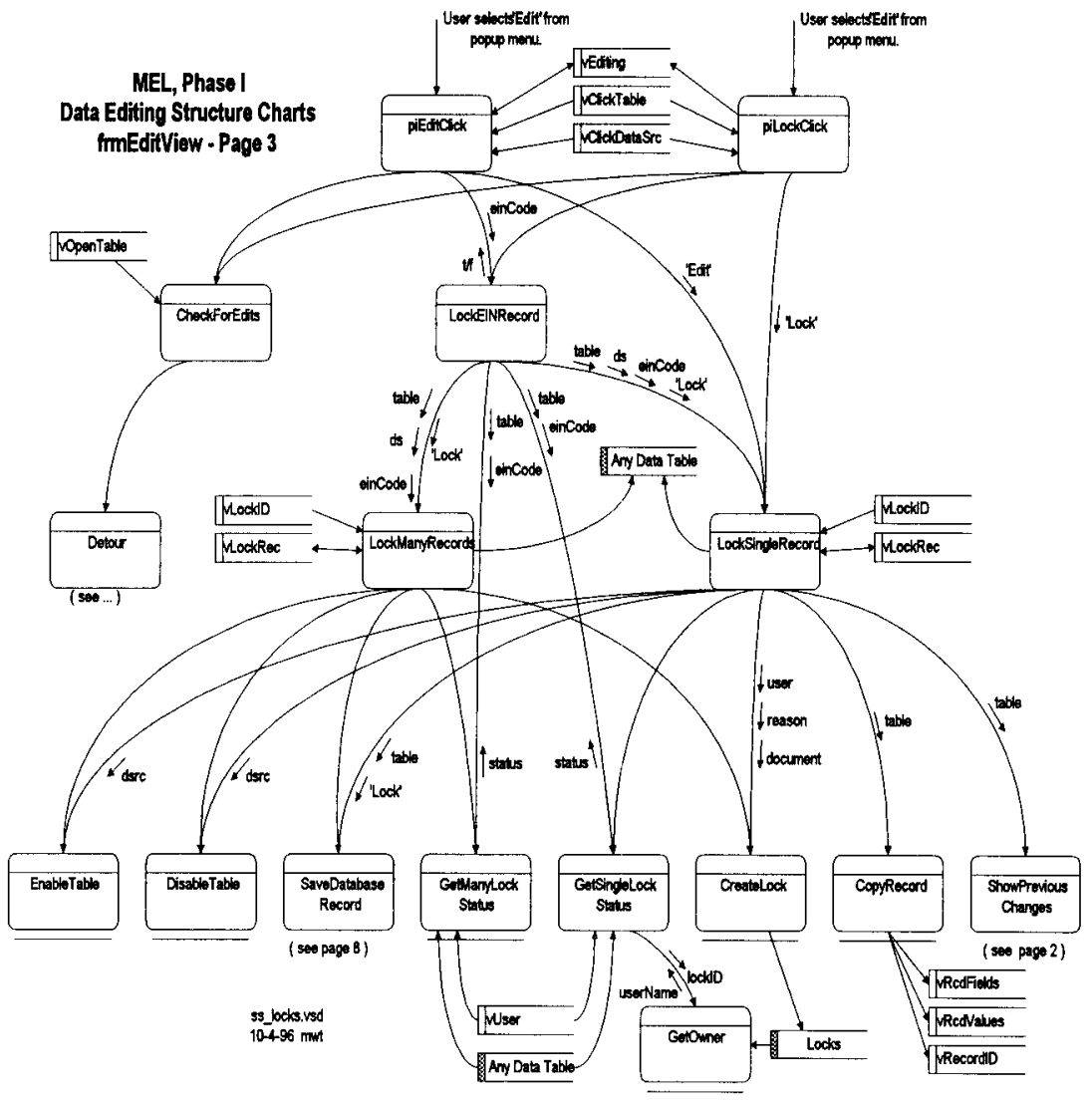


HNF-SD-WM-SDD-065

Revision 0

Page 73

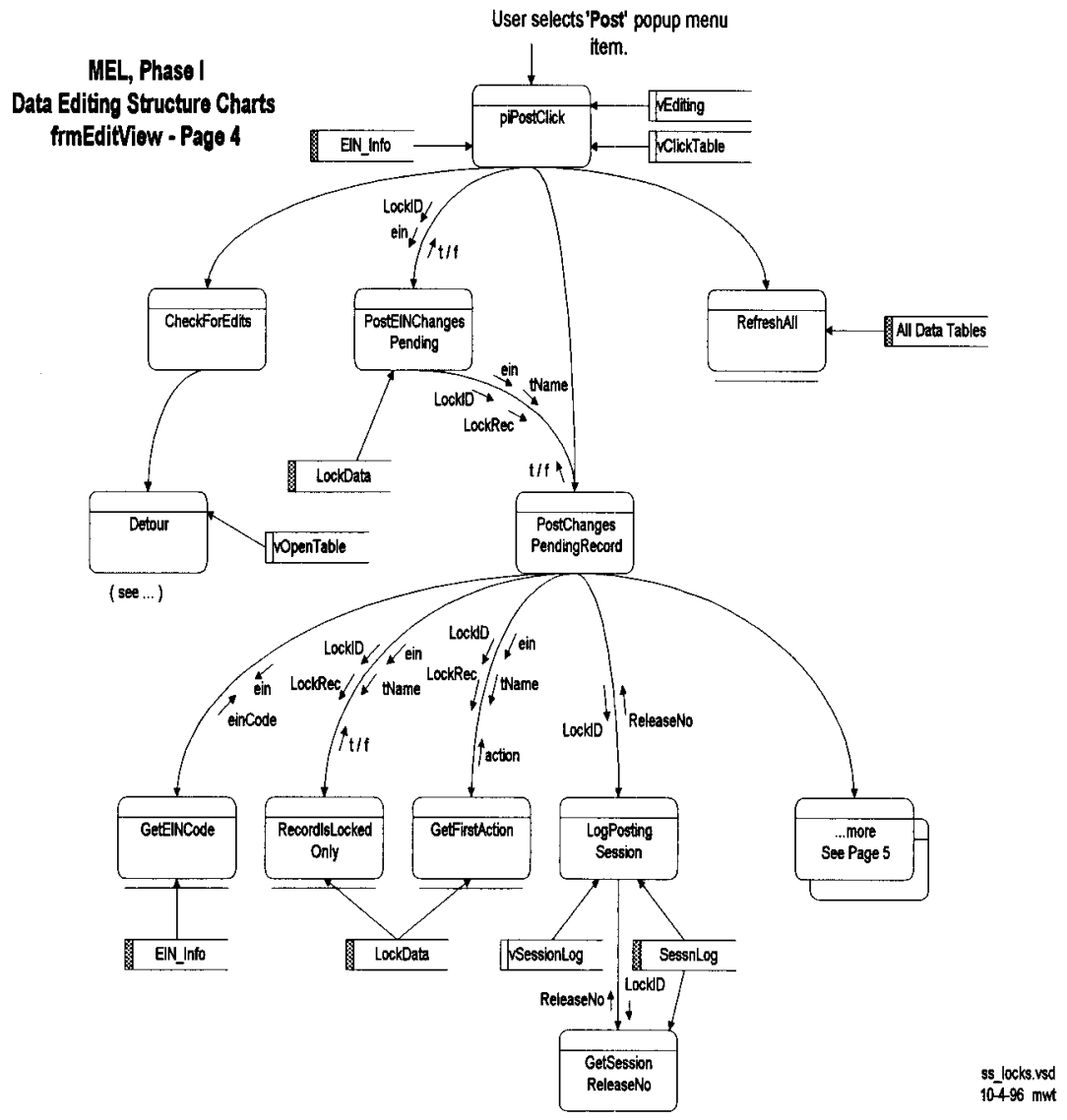




\section{MEL, Phase I}

\section{Data Editing Structure Charts \\ frmEditViow - Page 5}

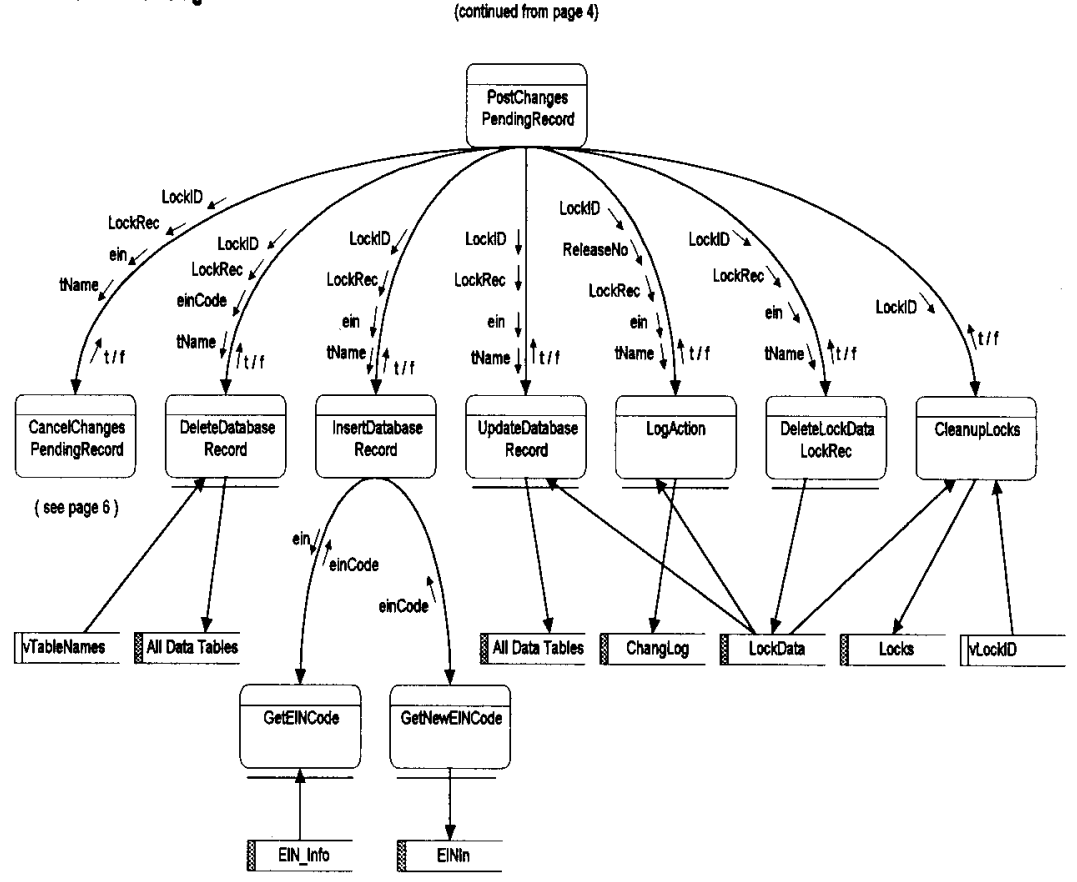


HNF-SD-WM-SDD-065

Revision 0

Page 75

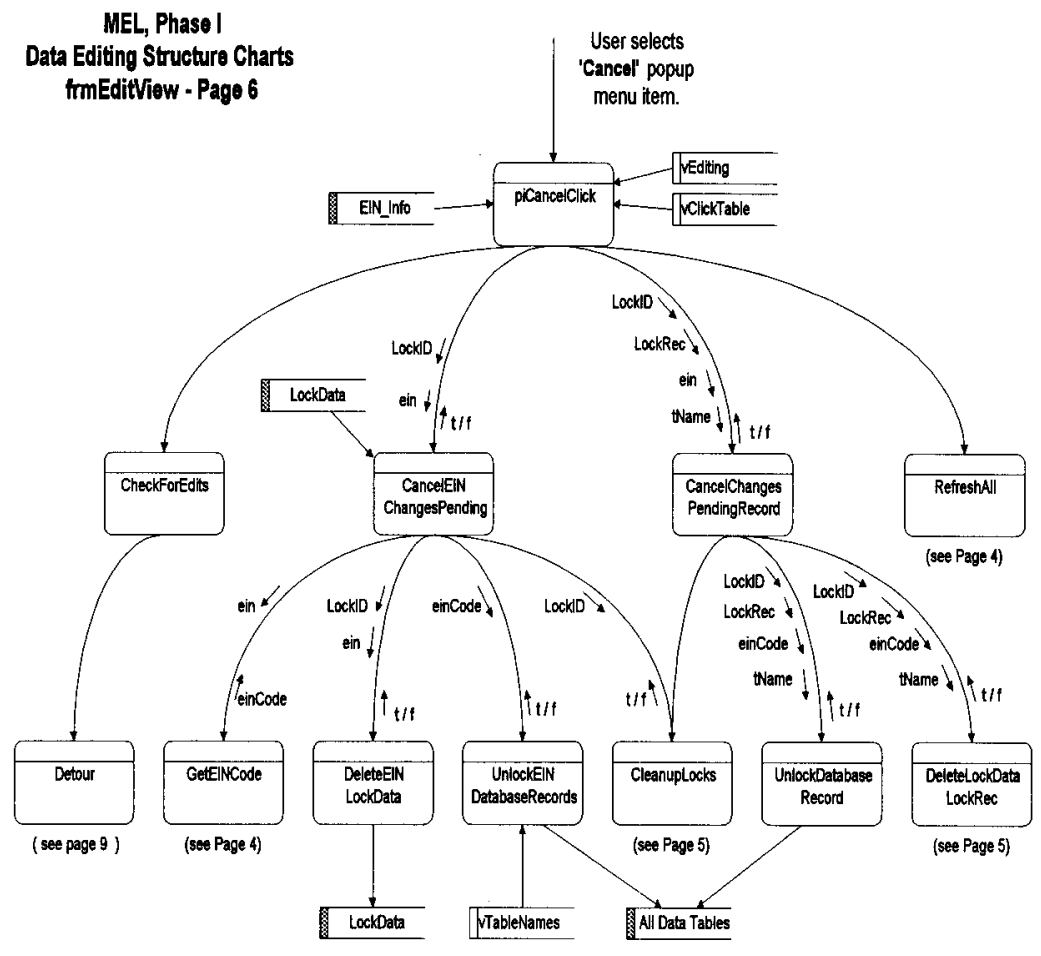


HNF-SD-WM-SDD-065

Revision 0

Page 76

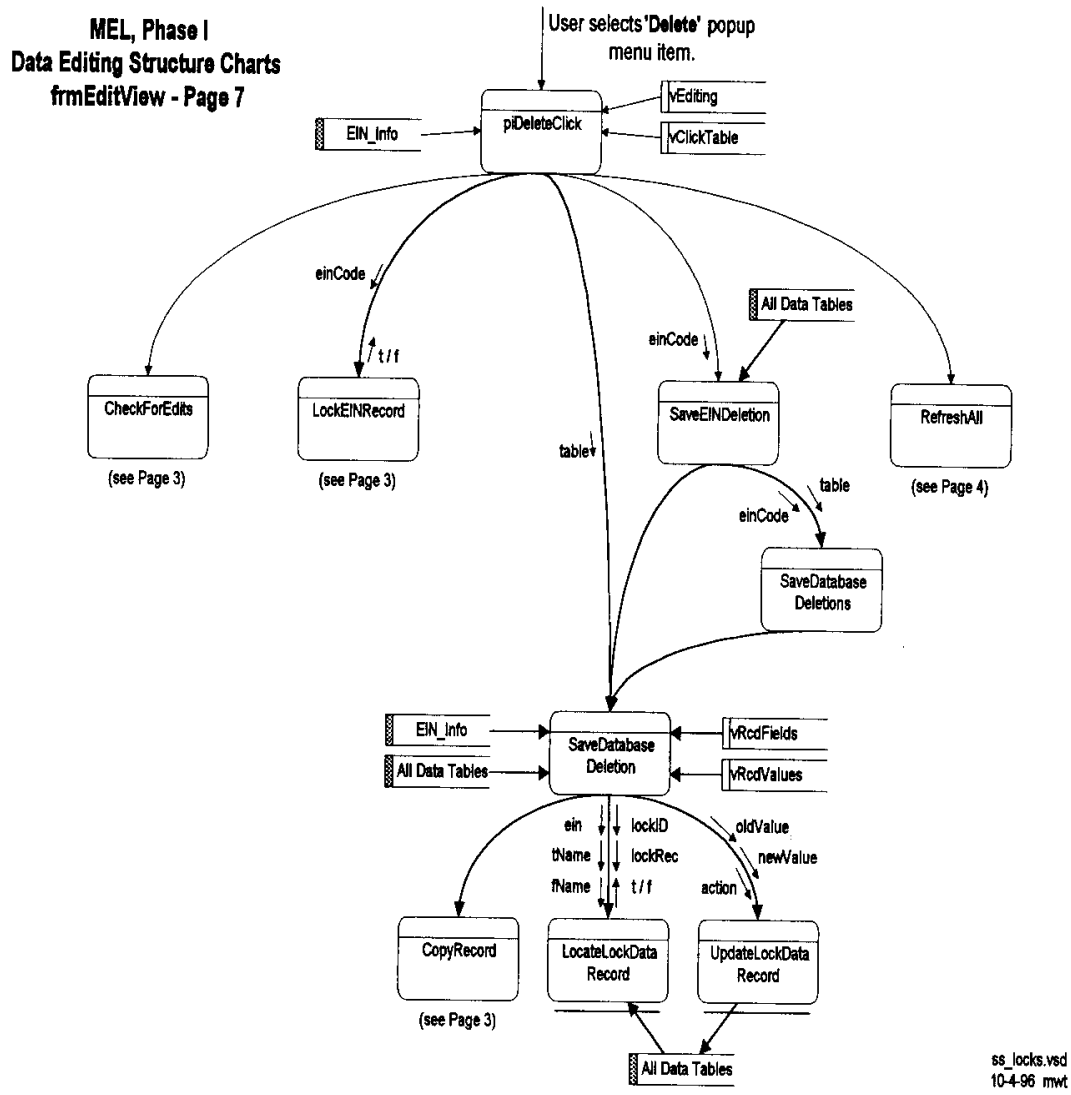


HNF-SD-WM-SDD-065

Revision 0

Page 77

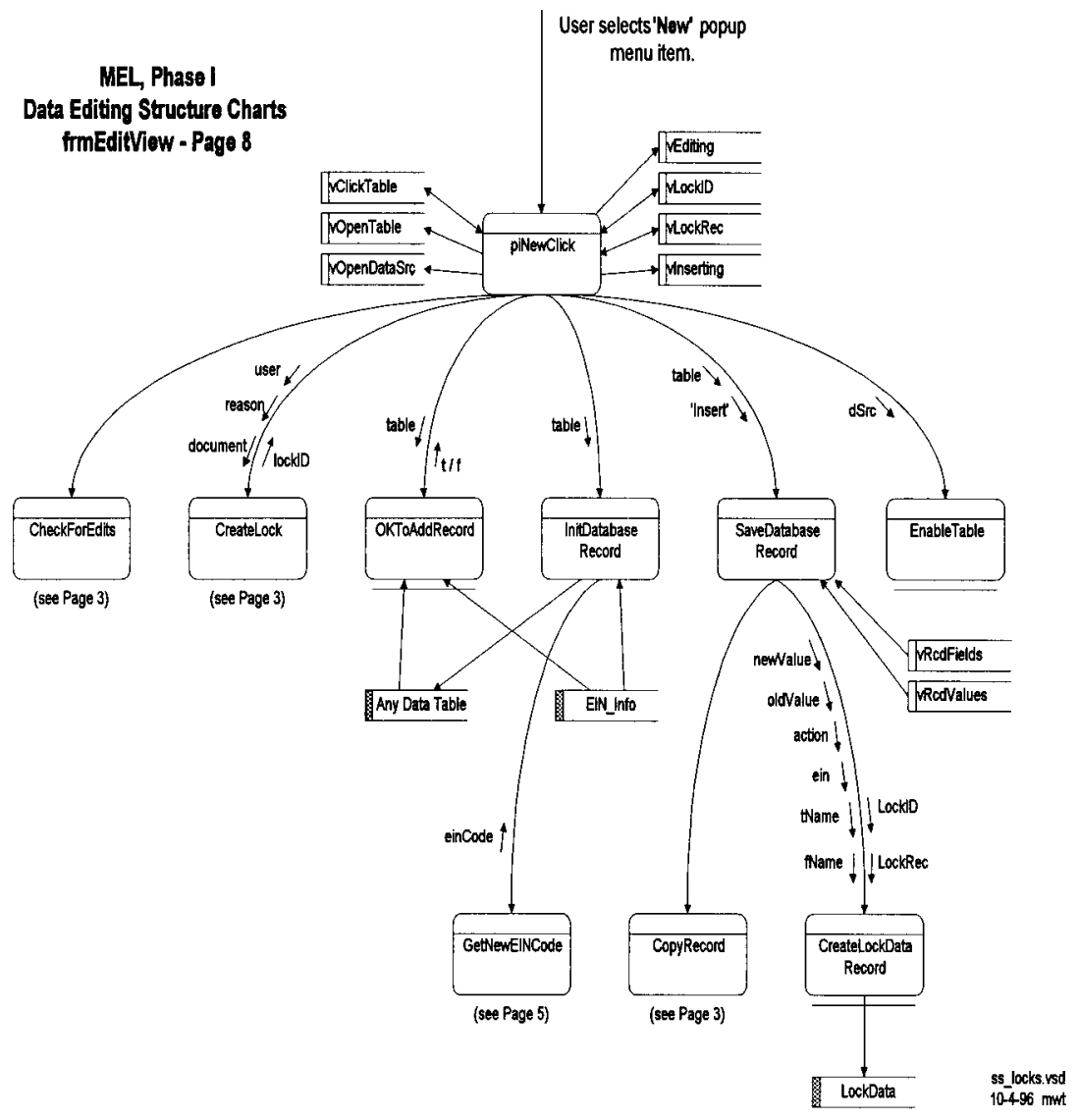


HNF-SD-WM-SDD-065

Revision 0

Page 78

MEL, Phase I

Data Editing Structure Charts

frmEditView - Page 9

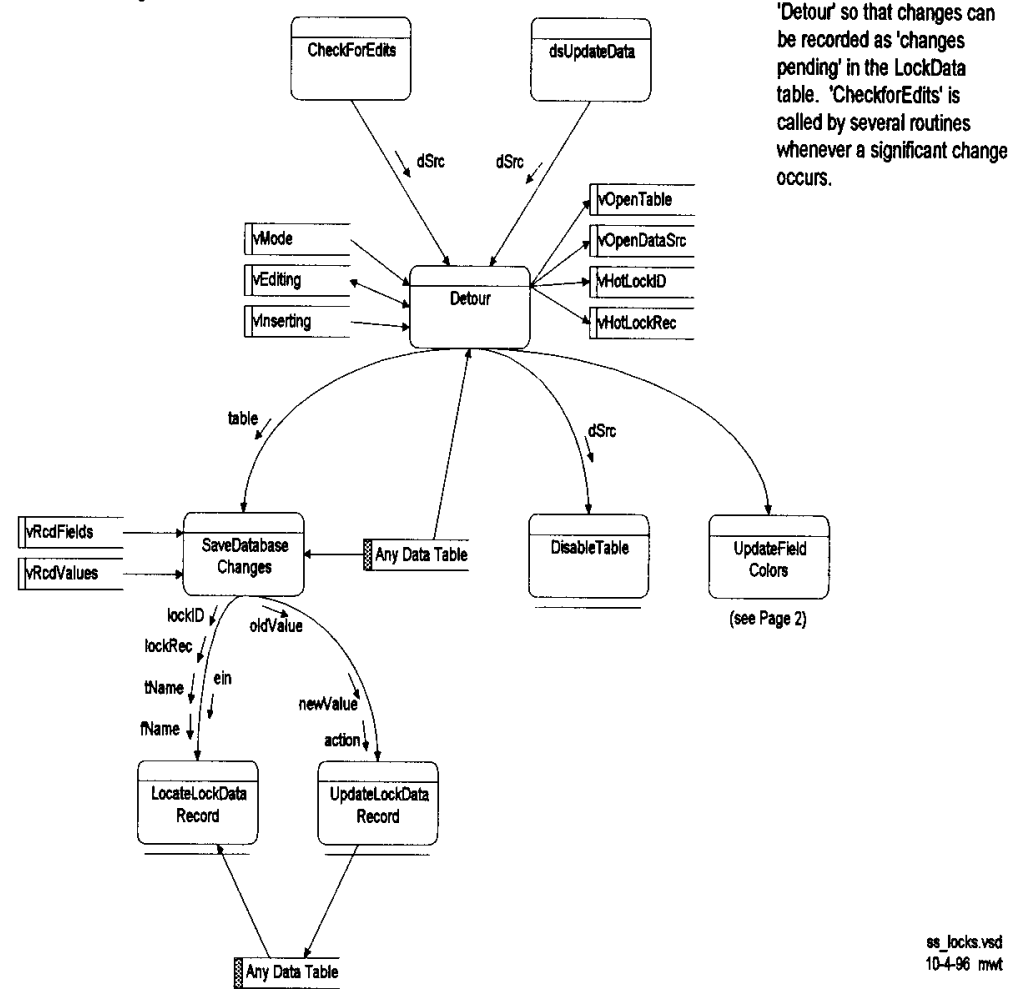

'ds UpdateData' is called whenever a database table is about to be updated. The update is intercepted by 'Detour' so that changes can be recorded as changes pending' in the LockData table. 'CheckforEdits' is whenever a significant change occurs. 
HNF-SD-WM-SDD-065

Revision 0

Page 79

\subsubsection{FOLDERS}

The MEL system divides its data into five major categories. These categories are represented in the application by "folders". A folder is a single page of data contained on a screen with a tab-like index feature. The folders in the MEL include Pointer, References, Nameplate, Specifications, and Safety. Each folder contains data grouped specific to these topics. 


\subsubsection{SCREENS}

The following sections contain pictures of the actual MEL user interface screens. Any data displayed is purely for example only.

\subsubsection{DATA VIEW - POINTER FOLDER}

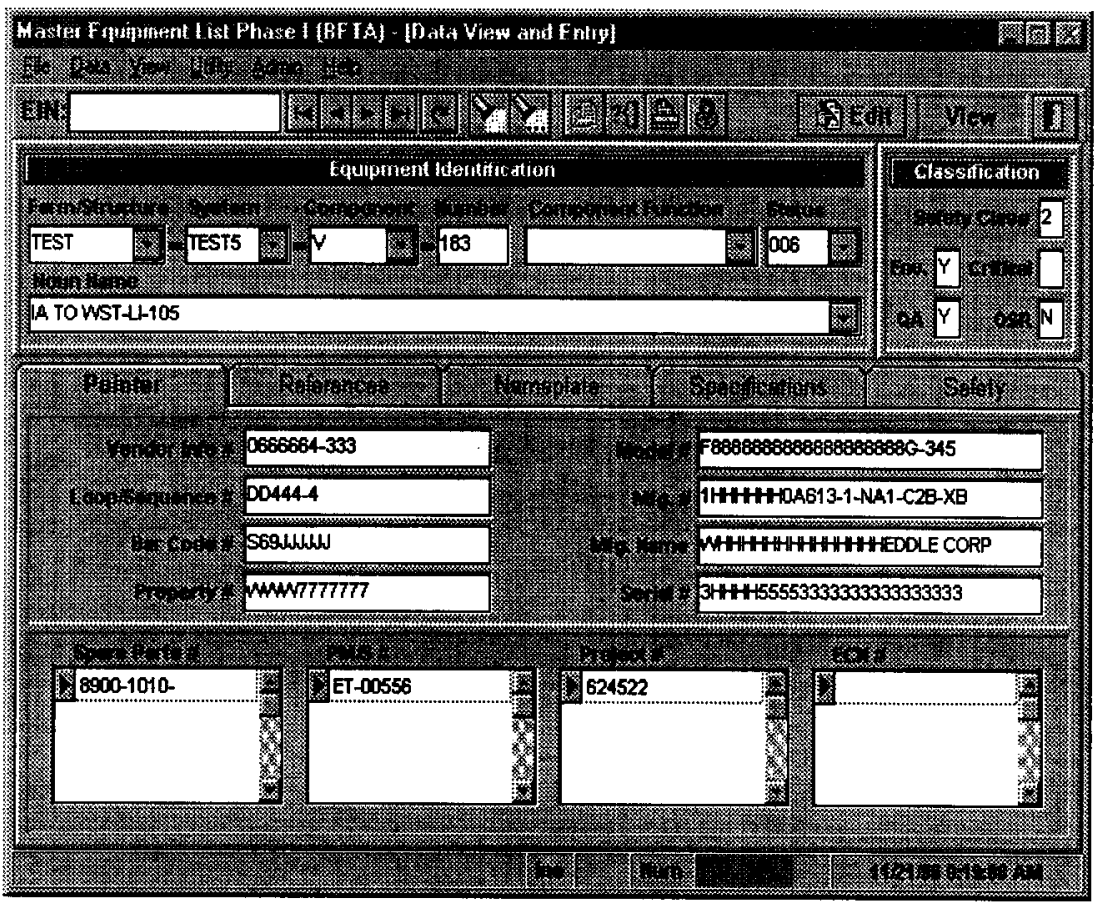


HNF-SD-WM-SDD-065

Revision 0

Page 81

\subsubsection{DATA VIEW - REFERENCES FOLDER}

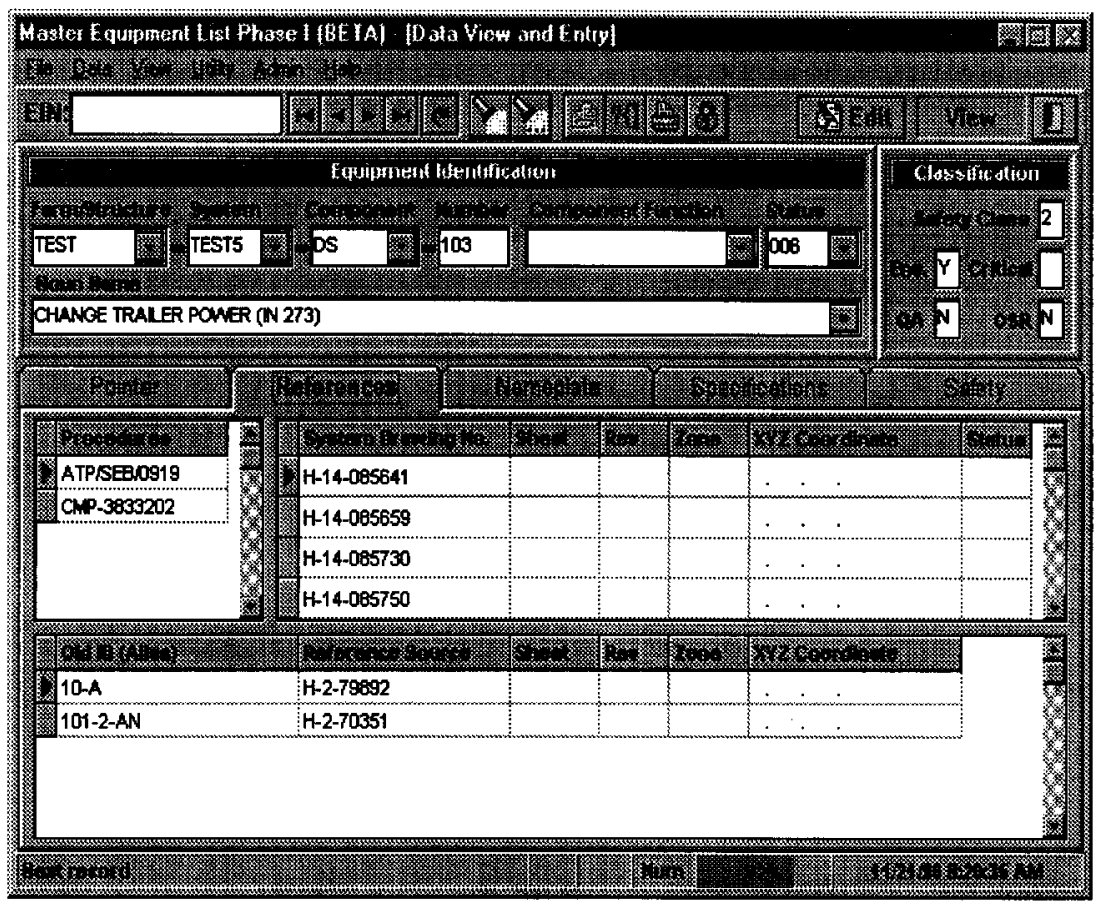


HNF-SD-WM-SDD-065

Revision 0

Page 82

\subsubsection{DATA VIEW - NAMEPLATE FOLDER}

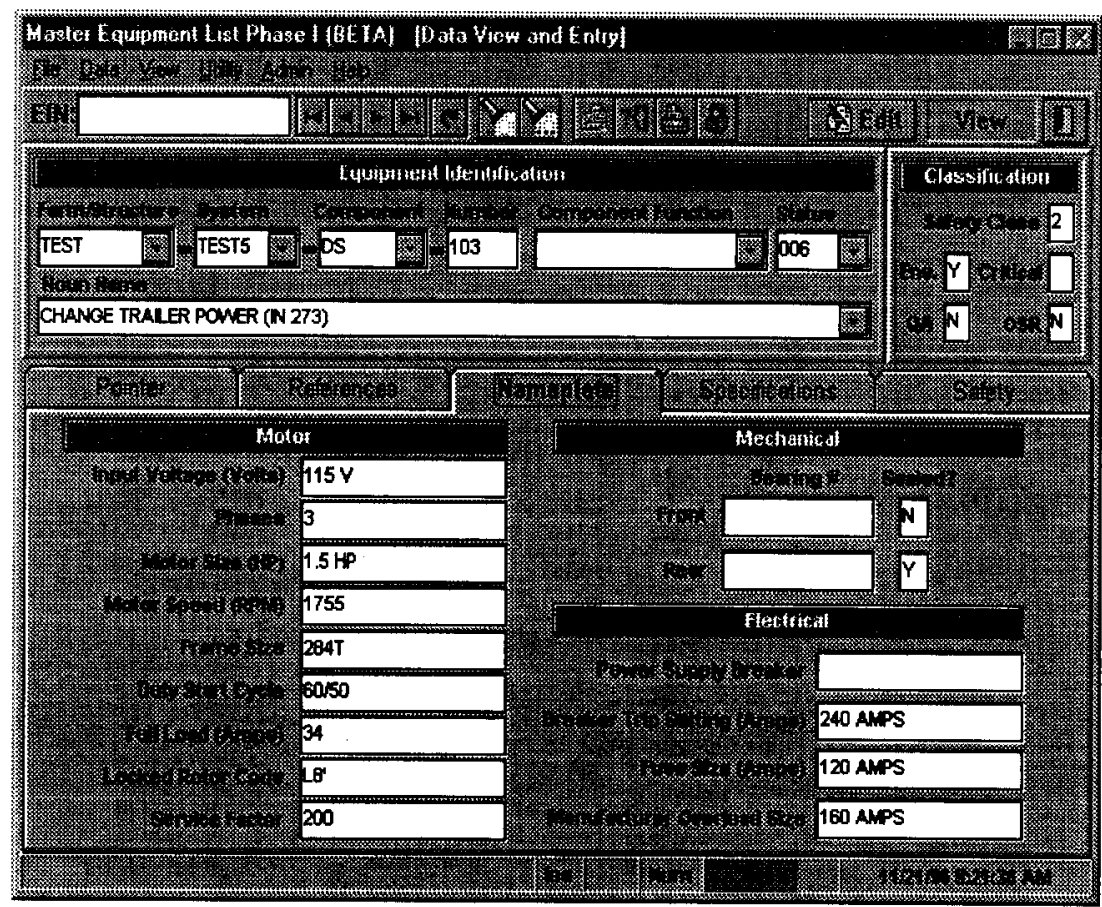


HNF-SD-WM-SDD-065

Revision 0

Page 83

\subsubsection{DATA VIEW - SPECIFICATIONS FOLDER}

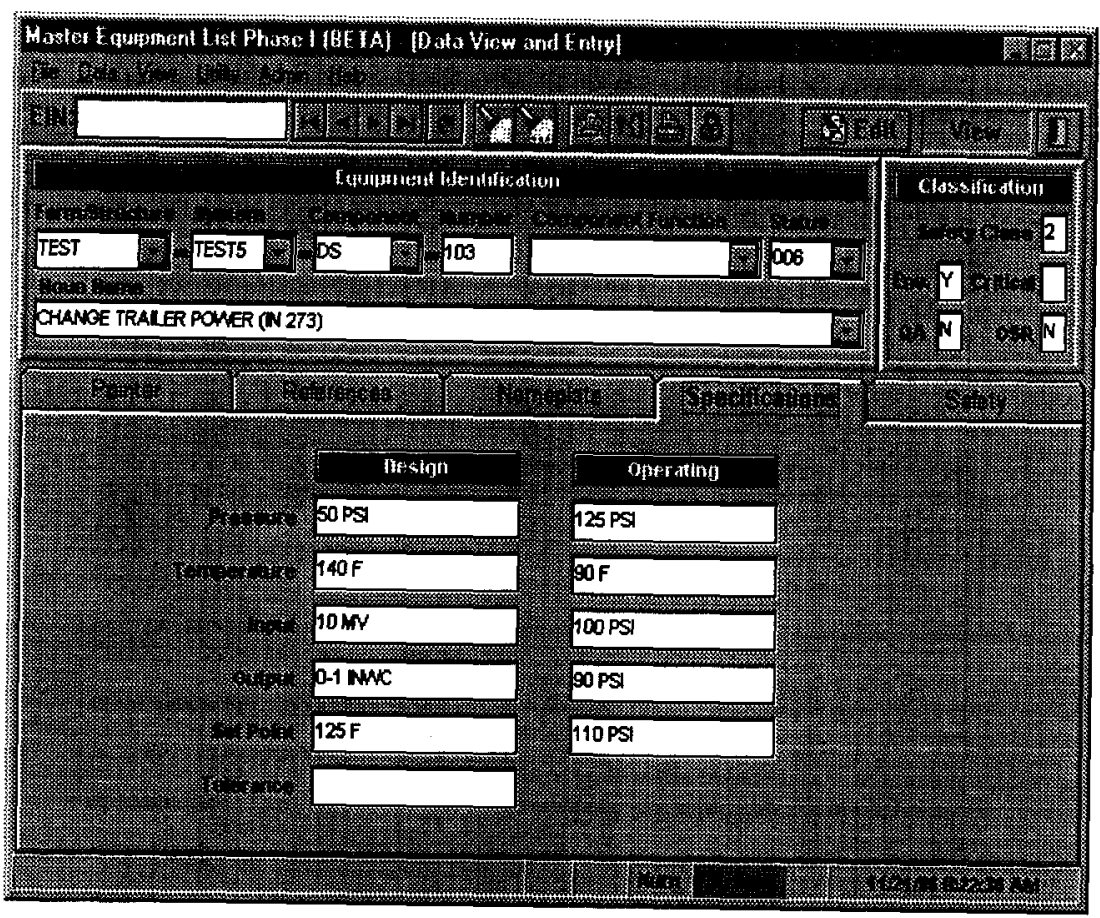


HNF-SD-WM-SDD-065

Revision 0

Page 84

\subsubsection{DATA VIEW - SAFETY FOLDER}

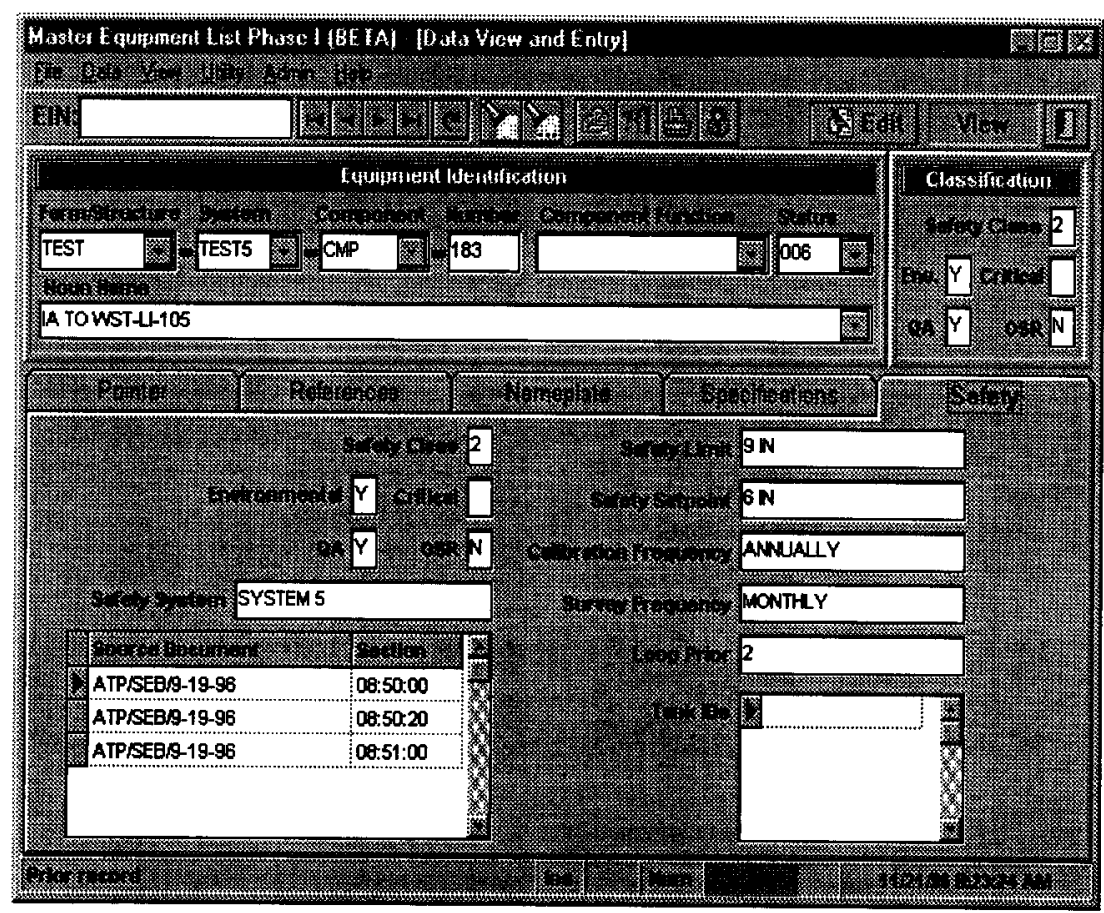




\subsubsection{QUERY}

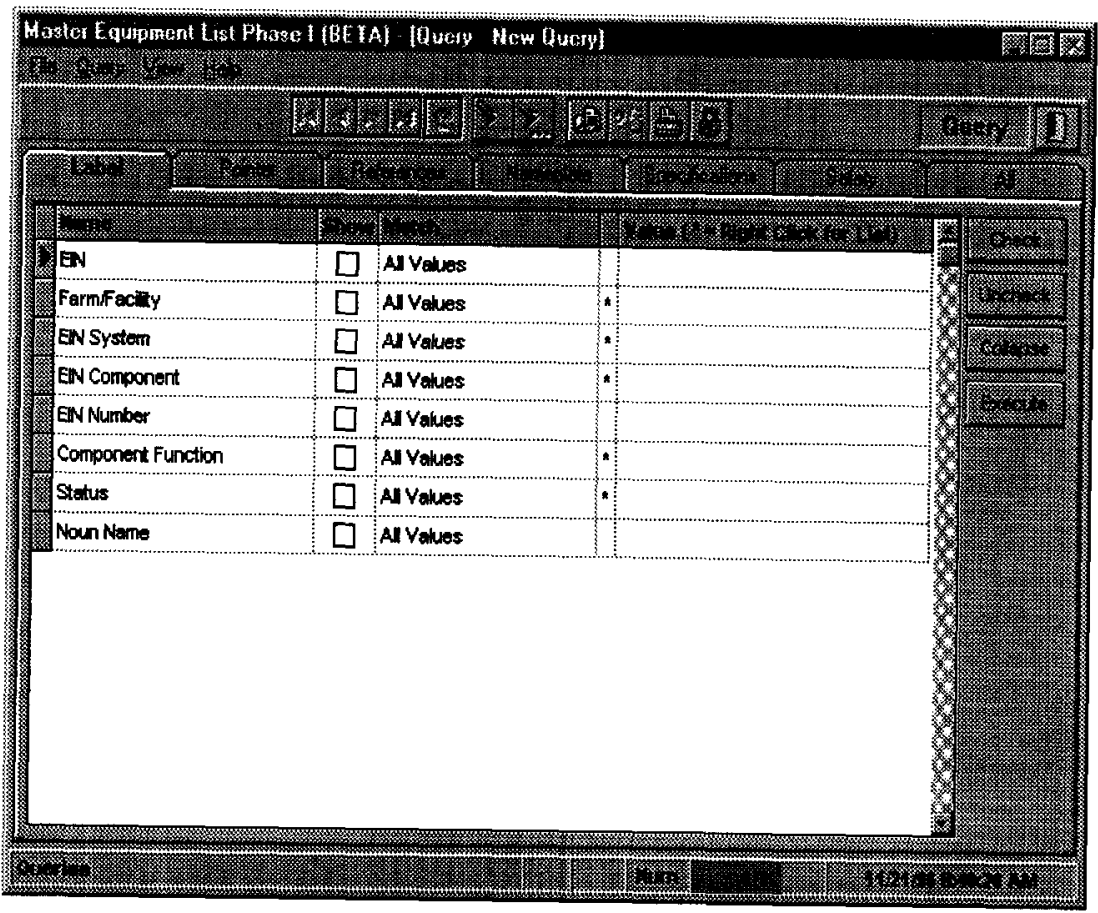


HNF-SD-WM-SDD-065

Revision 0

Page 86

\subsubsection{REPORTS}

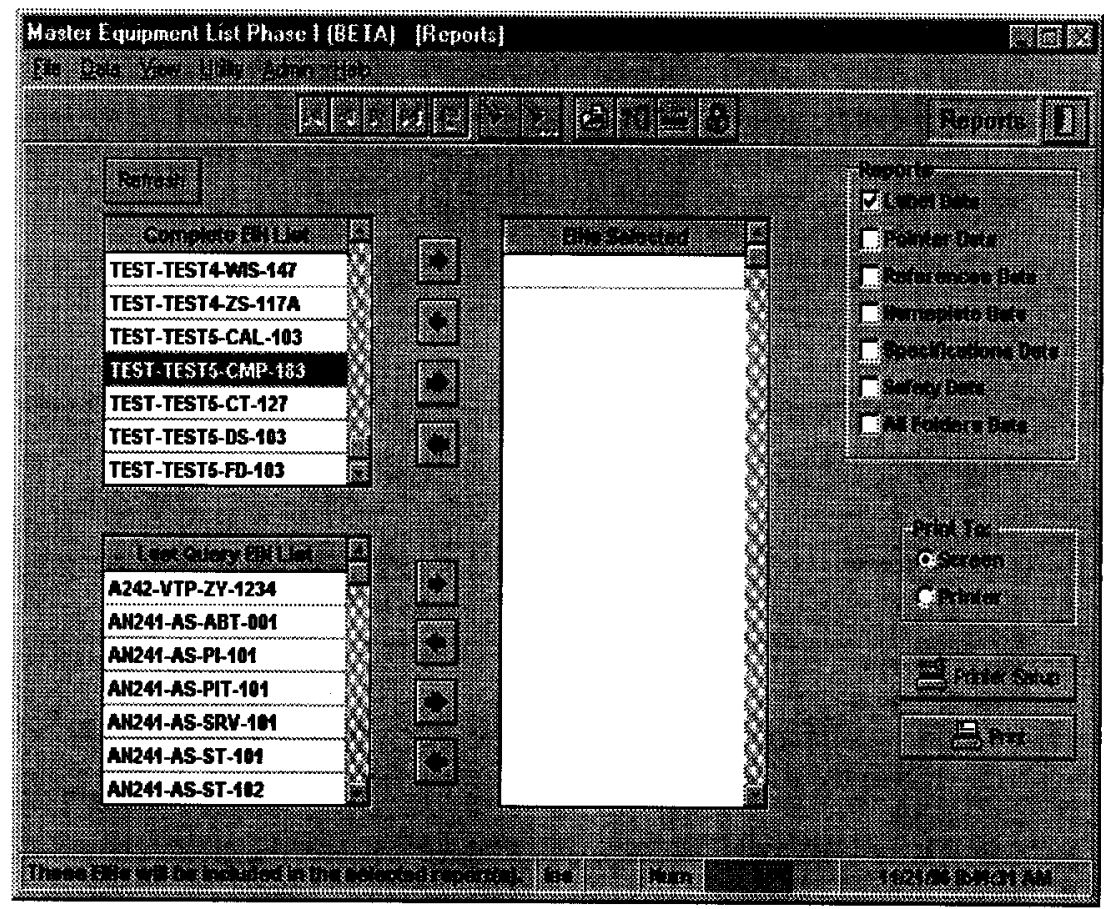


HNF-SD-WM-SDD-065

Revision 0

Page 87

\subsubsection{LOCKS AND CHANGES PENDING}

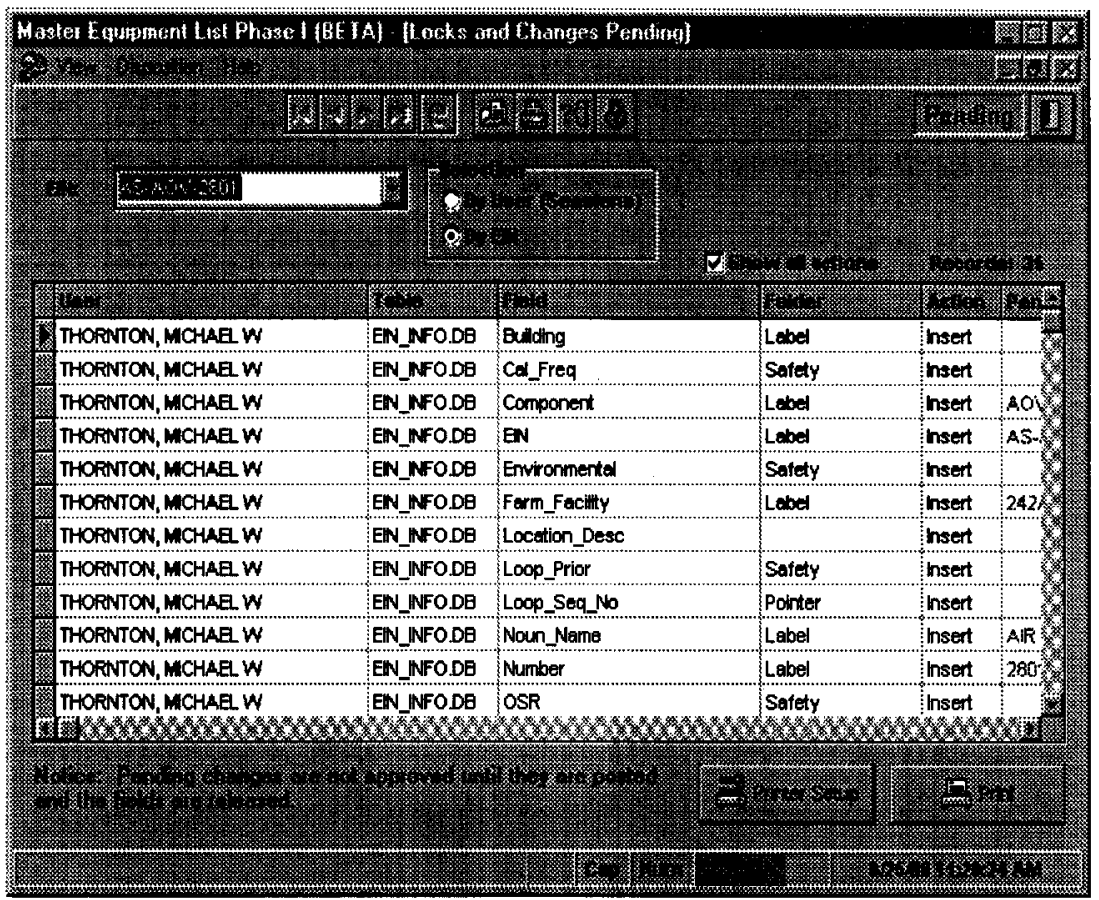


HNF-SD-WM-SDD-065

Revision 0

Page 88

\subsubsection{CREATE NEW EINS}

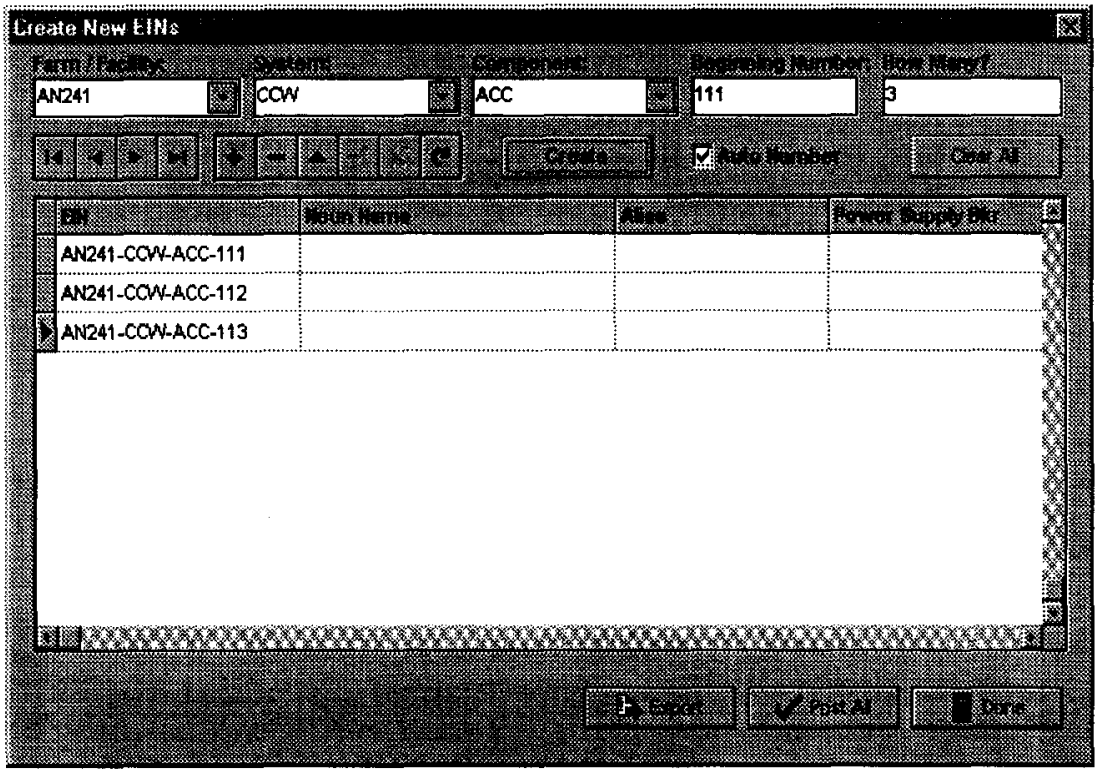


HNF-SD-WM-SDD-065

Revision 0

Page 89

\subsubsection{STANDARD DATA DEFINITIONS}

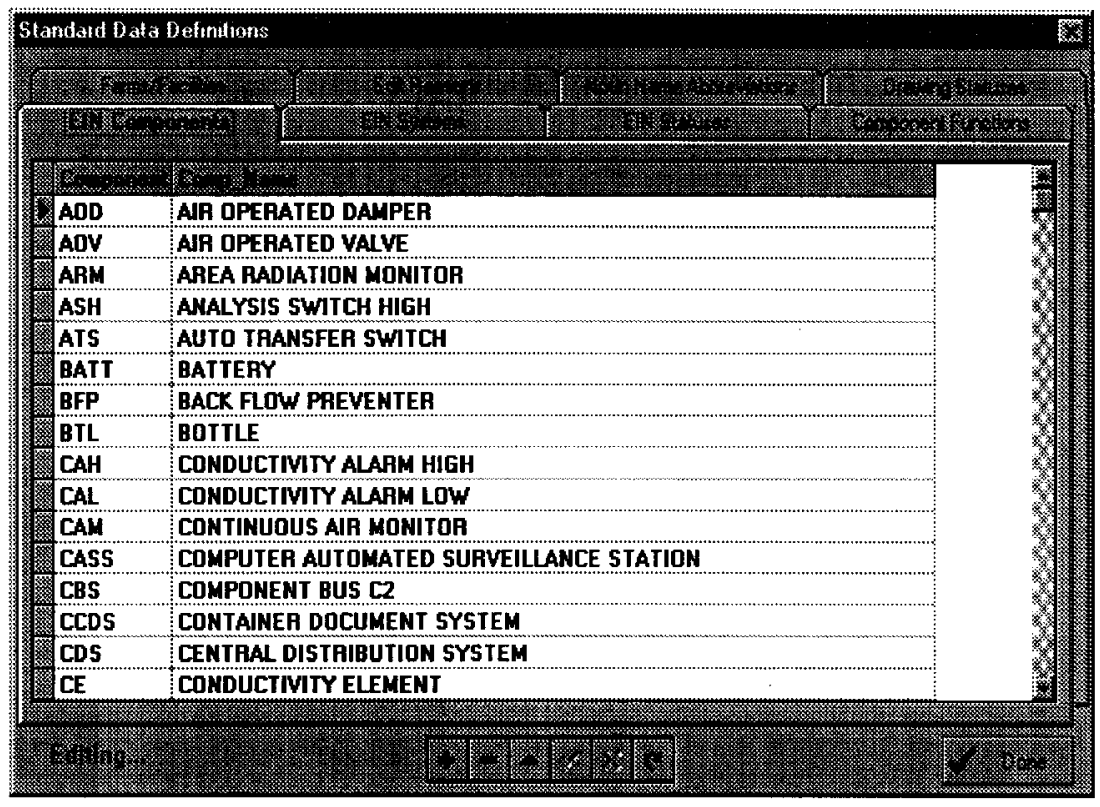




\subsubsection{CHANGE LOG}

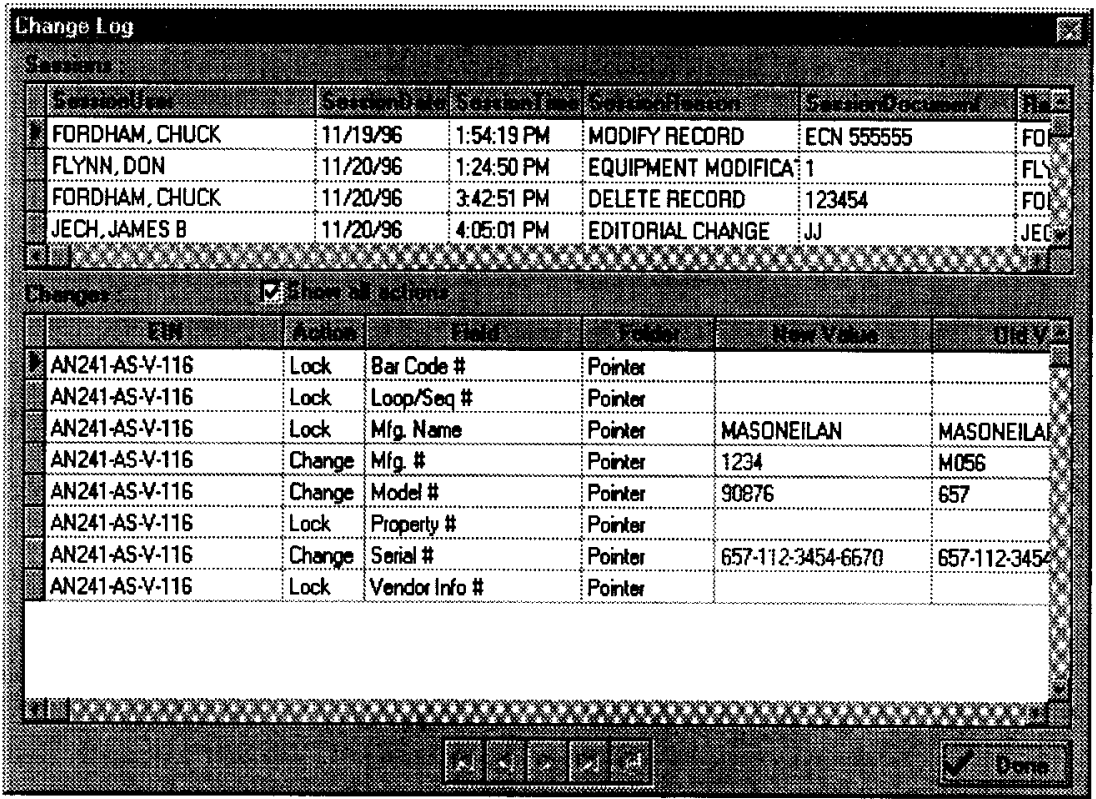


HNF-SD-WM-SDD-065

Revision 0

Page 91

\subsubsection{ACCESS CONTROL}

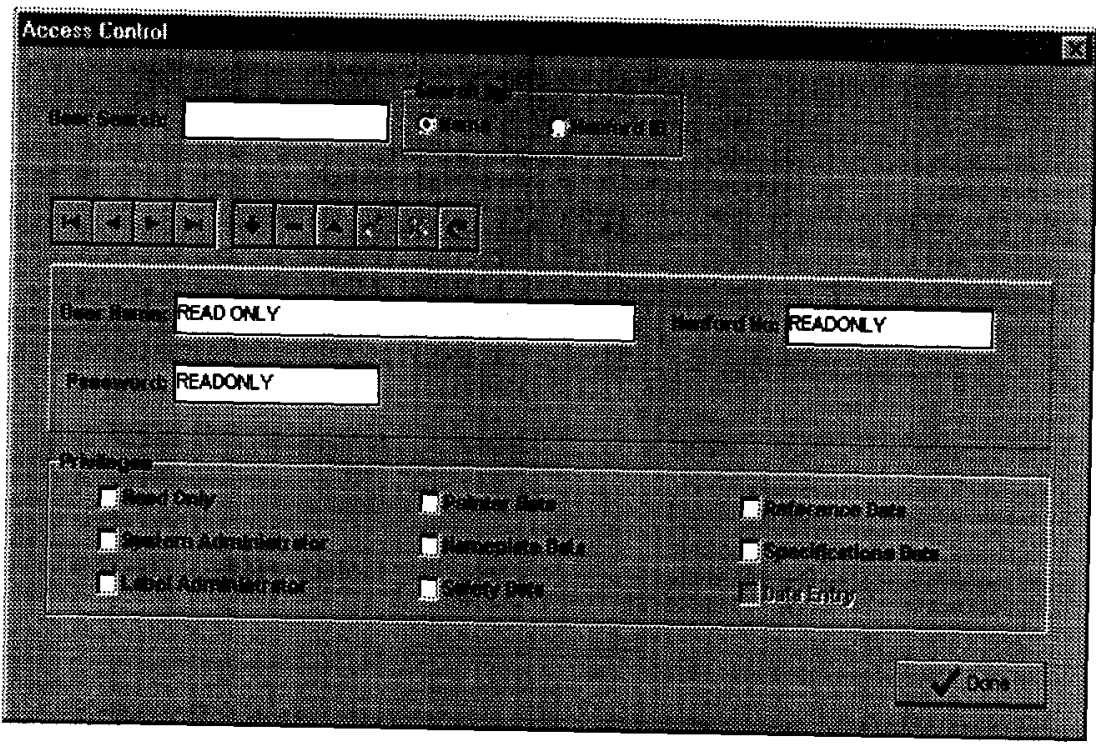


HNF-SD-WM-SDD-065

Revision 0

Page 92

\subsubsection{ABOUT}

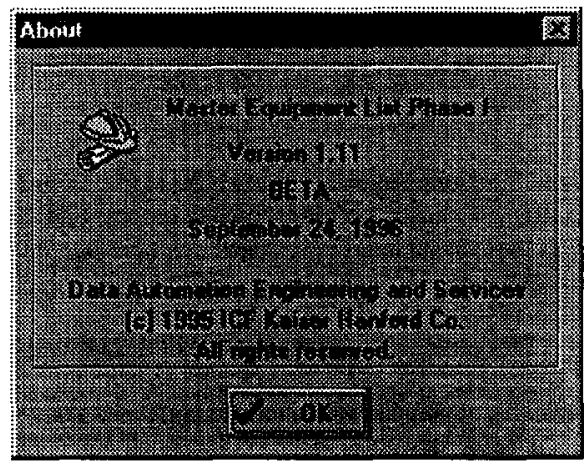


HNF-SD-WM-SDD-065

Revision 0

Page 93

\subsubsection{MENUS}

The following sections provide detail regarding the functionality of the MEL menus.

\subsubsection{MAIN MENU}

The MEL has several functions accessible through the menu aligned access the top of the MEL application main screen. This menu has the following options; File, Edit, Data, View, Utility, Admin.., and Help. These menu items have specific options available once the user accesses them. They are as follows:

File / Exit

- This option allows the user to exit the MEL application.

Edit / Find

- This option allows the user to perform a search for a specific EIN by a secondary field.

Edit / Find Next

- Allows the user to automatically search for the next value matching the previously defined search criteria.

Data / Data View - This function puts the database into view mode, posting any changes.

Data / Data Edit - This function puts the database into edit mode, prompting the user for an edit reason.

View / Folders

- This function allows the user the ability to view folder data.

View / Queries

- This function allows the user the ability to perform a query.

View / Reports

- This function allows the user the ability to print a report.

View / Locks

- This function allows the user the ability to view and disposition locked data, if privileges are applicable.

View / Hint Boxes - This function toggles the view of the yellow hint boxes providing user information when the mouse is held over certain fields.

Utility / Create EINs - This function allows the user to create new EINs. 
Utility / Definitions - This function allows the user to add, modify and remove master data definitions.

Utility / Change Log - This function allows the user to view the change log table.

Admin. / Access Control - This function allows the user to configure the MEL users list and access control.

Help / About _ - This function allows the user to view the release information regarding the current version of the MEL.

\subsubsection{QUERY MENU}

The menu on the "Query" page has the following options; File, Query, and Help. These menu items have specific options available once the user accesses them. They are as follows:

File / New Query _ - This option allows the user to create a new query.

File / Open Query... $\quad$ - This option allows the user to open an existing query.

File / Save Query _ - This option allows the user to save a query.

File / Save Query As... - This option allows the user to save a query with a different name.

File / Delete Query _ - This option allows the user to delete an existing query.

Query / Execute - This option allows the user to execute the current query.

Help - This option displays help regarding the use of the query page.

\subsubsection{LOCKS AND PENDING CHANGES MENU}

The menu on the "Locks and Pending Changes" page has the following options; View, Disposition, and Help. These menu items have specific options available once the user accesses them. They are as follows:

View / By User _ - This option allows the user to view locked data by user name. 
HNF-SD-WM-SDD-065

Revision 0

Page 95

View / By EINs

View / Show All
- This option allows the user to view locked data by EIN.

- This option allows the user to view all the data records, as opposed to only ones representing changes.

View / Cancel Session - This option allows the user to cancel the data for the selected session.

View / Cancel EIN - This option allows the user to cancel the data for the selected EIN.

View / Cancel User - This option allows the user to cancel the data for the selected user.

View / Cancel All - This option allows the user to cancel all the selected data.

View / Post Session - This option allows the user to post the data for the selected session.

View / Post EIN - This option allows the user to post the data for the selected EIN.

View / Post User - This option allows the user to post the data for the selected user.

View / Post All $\quad$ - This option allows the user to post all the selected data.

Help - This option displays help regarding the use of the query page.

\subsubsection{REPORTS}

The MEL Reports screen is built to accommodate several specific functions. First, the user may select to print data from one or many folders. Secondly, the user has the option to choose which EINs to report on by selecting them from a list. The user may also choose to print EINs generated from previously executed queries. Finally, the user may opt to print the report to the Screen or Printer.

The user may opt to print the following reports: Label Data, Pointer Data, References Data, Nameplate Data, Specifications Data, Safety Data, All Folders Data. Each report provides data from the respective folder. Report Examples are included in the following sections. 


\section{HNF-SD-WM-SDD-065 \\ Revision 0 \\ Page 96}

\subsubsection{LABEL DATA}

$9 / 25 / 96$

EIN

77Z-ALC-200 CAUSTIC

ZZZ-ALC-200 COND

ZZZ-ALC-200 FLUSH TK
Master Equipment List (Label Info)

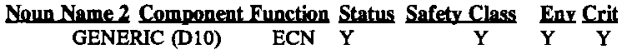

GENERIC (D10)

$001 Y$

$111 \mathrm{Y}$

QA OSR Label Code

Y Y

$\begin{array}{ll}\mathbf{Y} & \mathbf{Y} \\ \mathbf{Y} & \mathbf{Y}\end{array}$ 


\section{HNF-SD-WM-SDD-065 \\ Revision 0 \\ Page 97}

\subsubsection{POINTER DATA}

Farmifacility: AN FARM EIN: CHEME-VFD-100

Noun Name: CAUSTIC XFER PMP SPEED CONT

Component Function: VERTICAL Status: 006 Safety Class: 3 OSR: N

\section{Environmental: $Y \quad$ QA: $Y \quad$ Critical: $Y$}

Vendor Info
VFD

Bar Code No.

Property No. Model No

Mfg Veador No. Manufacturer

Serial No.

Spare Parts PM/S No. CAUSTIC XF 100

Project No.

FarmWacility: AN FARM

EIN: CHEMB-VFD-100

Component Function: VERTICAL

Noun Name: CAUSTIC XFER PMP SPEED CONT
Status: 006 Safety Class: 3
OSR: $N$
Environmental: $Y \quad$ QA: $Y \quad$ Critical: $Y$

Veador Info Looplseq Bar Code No. Property No. Model No Mfg Vendor No. Manufacturer Serial No. Spare Parts PM/S No. Project No.

FarmiFacility: AN FARM EIN: CHEMB-VFD-100 Component Function: VERTTCAL

Noun Name: CAUSTIC XFER PMP SPEED CONT
Status: 006 Safety Class: 3
OSR: $\mathbf{N}$
Environmental: Y
QA: Y
Critical: Y

Vendor Info f Loplseq Bar Code No. Property No. Model No Mfg Vendor No. Manufacturer Serial No. Spare Parts PM/S No. Project No. 


\subsubsection{REFERENCE DATA}

$9 / 25 / 96$

Master Equipment List (Reference Info)

Page 1
FarmlFacility: AN FARM $\quad$ EIN: CHEMB-VFD-100 Component Function: VERTICAL
Noun Name: CAUSTIC XFER PMP SPEED CONT

Status: 006 Safety Class: 3 OSR: $N \quad$ Environmental: $Y \quad$ QA: $Y \quad$ Critical: $Y$

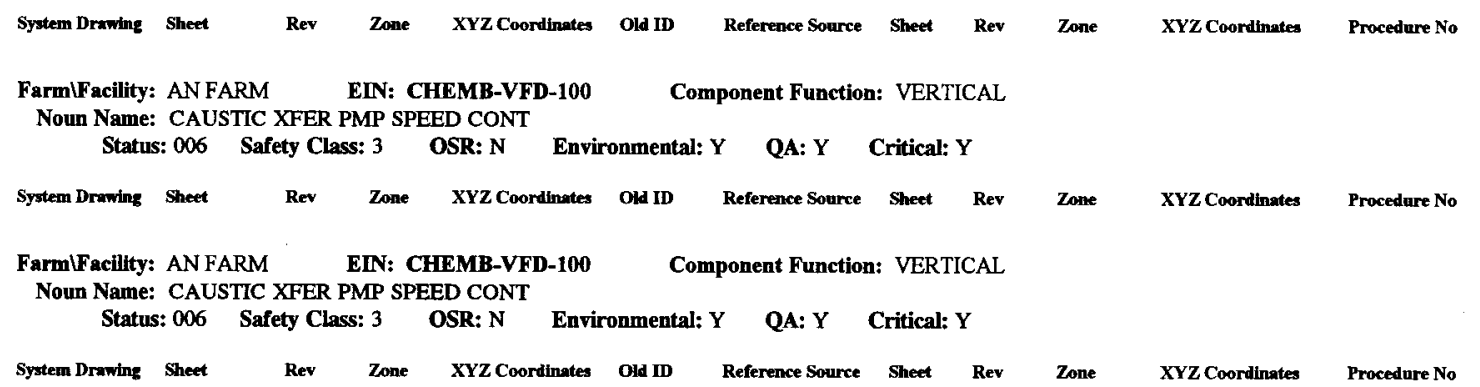



HNF-SD-WM-SDD-065

Revision 0

Page 99

\subsubsection{NAMEPLATE DATA}

$9 / 25 / 96$

Master Equipment List (Nameplate Info)

Page I

EIN: CHEMB-VFD-100

FarmUFacility: AN FARM

Noun Name: CAUSTIC XFER PMP SPEED CONT

Status: 006 Safety Class: 3 OSR: N
Component Function: VERTICAL

Environmental: $Y \quad$ QA: $Y \quad$ Critical: $Y$

Motor

Mechanical

Imput Voltage (Volts):

Phases:

Motor Size:

Motor Speed (RPM): 4

Frame Size:

Duty Start Cycle:

Full Load (Amps): 7

Locked Rotor Code: 8

Service Factor: 9
1

Front: 10

Rear: 11

Electrical

6
Bearings No.

$\mathbf{Y}$

N

Sealed?

Power Supply Breaker: 12
$\begin{aligned} & \text { Breaker Trip Setting (Amps): } \\ & \text { Fuse Size (Amps): } \quad 3\end{aligned}$
Manufacturer Overload Size:


HNF-SD-WM-SDD-065

Revision 0

Page 100

\subsubsection{SPECIFICATION DATA}

$9 / 25 / 96$

Master Equipment List (Specification Info)

Page 1

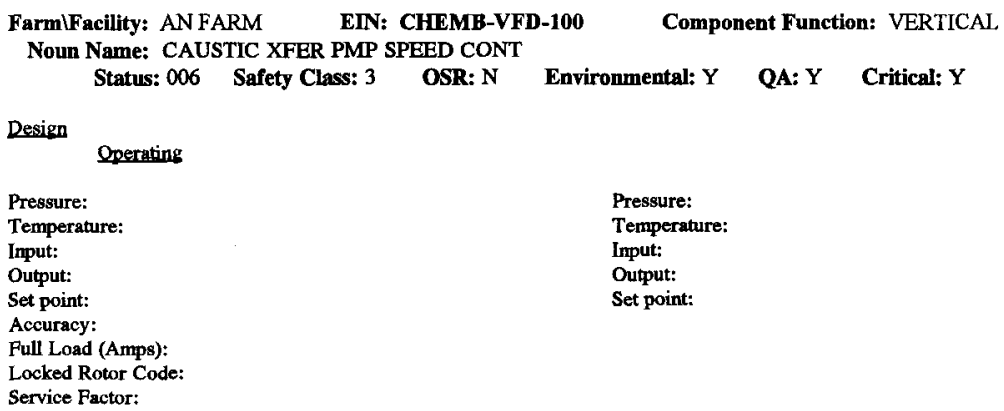




\subsubsection{SAFETY DATA}

\section{FarmUFacility: AN FARM EIN: CHEMB-VFD-100 Component Function: VERTICAL \\ Noun Name: CAUSTIC XFER PMP SPEED CONT}

Status: 006 Safety Class: 3 OSR: $N$ Environmental: $Y$ QA: $Y$ Critical: $Y$

$\begin{array}{lllllll}\text { Safety Set potnt } & \text { Safety Limit } & \text { Safety System } & \text { Sarvey Freq. } & \text { Cal Freq. } & \text { Loop Priority } & \text { Safety Source Document } \\ 1 & 1 & 1 & 1 & 1 & \text { Section } & 111111\end{array}$

FarmLFacility: AN FARM CIN: CHEMB-VFD-100 Component Function: VERTICAL

Noun Name: CAUSTIC XFER PMP SPEED CONT

Status: 006 Safety Class: 3 OSR: $N$ Environmental: $Y$ QA: $Y$ Critical: $Y$

Safety Set point Safety Limit Sufety System Survey Freq. Cal Freq. Loop Priority Safety Source Document Section $\quad$ Tank ID

FarmUFacility: AN FARM EIN: CHEMB-VFD-100 Component Function: VERTICAL

Noun Name: CAUSTIC XFER PMP SPEED CONT

Status: 006 Safety Class: 3 OSR: $N$ Environmental: $Y \quad$ QA: $Y \quad$ Critical: $Y$

\begin{tabular}{|c|c|c|c|c|c|c|c|}
\hline $\begin{array}{l}\text { Sufety Set point } \\
1\end{array}$ & $\begin{array}{l}\text { Safety Limit } \\
1\end{array}$ & $\begin{array}{l}\text { Safety System } \\
1\end{array}$ & $\begin{array}{l}\text { Survey Freq. } \\
1\end{array}$ & $\begin{array}{l}\text { Coll Freq. } \\
1\end{array}$ & $\begin{array}{l}\text { Loop Prlority } \\
1\end{array}$ & Safety Source Document & $\begin{array}{l}\text { Section } \\
111111\end{array}$ \\
\hline
\end{tabular}





\subsubsection{PRIVILEGES}

Every MEL user has a set of pre-defined privileges. This information is stored in the user table (user.db). Each record in this table includes the user's name, Hanford ID, password, and privilege. The privilege field is a string of eight characters, in which each individual character represents access to a different data group. Each character is either a " 0 " or a " 1 ", where a " 0 " represents no access, and a " 1 " allows access. The data groups are represented as follows:

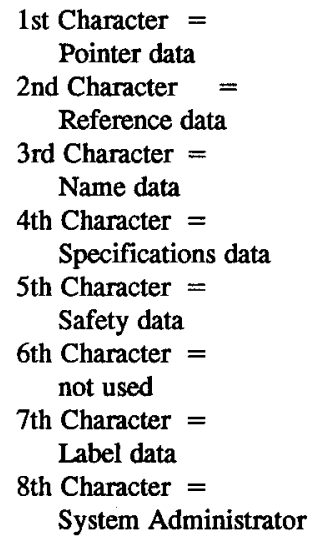

\subsubsection{ACCESS CONTROL}

Access to the MEL system and its different functions are controlled according to predefined privileges. These privileges are setup and maintained by the system administrator (see the "Privileges" section for more information).

Access for a user depends on the privilege code, which is applied to the MEL system. Users only have write access to those data groups of the application which are represented in their respective privilege code. Any data group in which access is not allowed is automatically set to read-only. The privilege code is checked after user logon, and write access to the different parts of the system are set at that time. 
HNF-SD-WM-SDD-065

Revision 0

Page 103

\subsubsection{ChANGES PENDING \& CHANGE LOG}

\subsection{LAUNCHER}

The MEL system contains an executable file which is used to start or "launch" the main program. This file is needed not only to start the client application, but to handle network connections as well.

Since the MEL is a multiple user database, the data must be centrally located on a common fileserver. Connections to this fileserver must occur prior to starting the MEL. Furthermore, these connections need to be closed once the user terminates the MEL. These tasks are all accomplished by the MEL launcher application.

The MEL launcher has settings configured within an initialization (INI) file to launch a specified version of the application. This INI file is communicated to the launcher on the Windows command line. For example, "C:IWINAPPSIMELILAUNCH.EXE MELBETA.INI" would tell the launcher to initiate the beta version of the MEL.

After determination of the version, the launcher attempts to make a connection to every fileserver that the MEL requires. If these connections cannot be made, the user is notified and the session is terminated. Otherwise, the MEL application is initiated. Once the MEL has been started, the launcher waits in the background until the user terminates the session, at this point, the launcher disconnects the MEL network connections and returns the user to the Windows environment. The DAES Launcher Application User Manual can be found in the DAES technical library.

\subsection{DISTRIBUTION / INSTALLATION}

\subsubsection{RELEASE AREAS \& VERSIONS}

The MEL system has several release areas associated with it. These include Alpha, Beta, Alt1, and Production. They are defined as follows:

Alpha Release Area - This area contains the most recent development work. The source code is untested and contains data which may be discarded at any time. It is for developer's use only, and available only to selected users for testing proposes. 
Beta Release Area

Alt1 Release Area
- This area contains the version of the MEL that is currently in the process of acceptance testing. Source code for this area must be kept under configuration control, with a copy being maintained by the software custodian. Access to this area is available to selected beta testers only.

- This area is a temporary holding area for a tested copy of the MEL application. In this area, the application has been acceptance tested, however, the system may still need data transferred in from other sources, or may need operational testing. Data in this area will not be removed without the system administrators confirmation.

Production Release Area - This area houses the production version of the MEL. This is the area in which the MEL is used by only authorized users and data is strictly maintained. There should be no testing in this area, since the data is quality affecting.

Besides having separate release areas, each area may have a different version of the MEL. For example the Production Release Area might contain Version 1.0. However, the Beta Release Area might have Version 2.0, and the Alpha Release Area might be on Version 3.0. Nevertheless, a user may always find out what release and version they are current running by looking in the Help / About section of the application.

\subsubsection{INSTALLATION}

The Installation process for the MEL system involves several functions. First, the installation program or "Installer" is a third-party software package called "WINStall" developed by OnDemand software. This program, which is the Hanford Site standard for network installations, places files from a network fileserver to a location on the client machine. These files and subdirectories include:

$\begin{array}{ll}\text { MEL_PHS1.EXE } & \text { - MEL executable file } \\ \text { LAUNCH.EXE } & \text { - MEL Launcher } \\ \text { MEL*.INI } & \text { - MEL Initialization file } \\ \text { MEL1*.VER } & \text { - MEL Version File } \\ \text { MEL.ICO } & \text { - MEI Icon } \\ \text { LOC_DATA subdirectory } & \text { - Subdirectory for temporary files, reports, local tables } \\ \text { PREFS subdirectory } & \text { - Subdirectory for user preferences }\end{array}$


HNF-SD-WM-SDD-065

Revision 0

Page 105

All files are located on the client machine in the directory C:IWINAPPSIMEL/RELEASE NAME, where release name is the name of the installed release. The MEL1*.VER file depends on the installed release. The * character is replaced with a representation of the release type.

Each release contains a *.ver file which is used for version identification. If a user has not installed the most recent version of a release, they are prompted to install the new version before continuing. The comparison is performed by using the date and time stamp of the *.ver files, with the most recent one being available on the network fileserver.

\subsubsection{USER COMPUTER RESOURCE REQUIREMENTS}

The following configuration is recommended for user computers:

- 486 or higher IBM-Compatible Personal Computer (PC).

- A hard disk drive configured as " $\mathrm{C}:$ ".

- At least 8 megabytes of RAM, 16 megabytes is recommended.

- At least 30 megabytes free disk space on "C:".

- Hanford End System Operating Environment (ESOE). 
HNF-SD-WM-SDD-065

Revision 0

Page 106

\subsection{REFERENCES}

1) WHC-CM-6-1, Standard Engineering_Practices.

EP-5.8, Rev. 3, "Engineering Document Content Guidelines", Exhibit 12, February 7, 1992.

2) WHC-CM-4-2, Quality Assurance Manual.

QR-19.0, Rev. 0, "Software Quality Assurance Requirements," December 6, 1991.

3) WHC-SD-WM-CSRS-023, System Requirements Specification for Master Equipment List Phase I, April, 1995.

4) WHC-SD-WM-SDP-012, System Development Plan for Master Equipment List Phase I, September, 1995.

6) WHC-CM-3-10, Software Practices, September 30, 1996.

7) WHC-CM-3-5, Document Control and Records Management Manual, April 10, 1996. 
THIS PAGE INTENTIONALLY LEFT BLANK 